\title{
Application of cylindrical IE-GSTC to physical metasurfaces
}

This paper was downloaded from TechRxiv (https://www.techrxiv.org).

\section{LICENSE}

CC BY 4.0

SUBMISSION DATE / POSTED DATE

$25-01-2021 / 01-11-2021$

\section{CITATION}

Sandeep, Srikumar; Gasiewski, Albin; Peterson, Andrew F (2021): Application of cylindrical IE-GSTC to physical metasurfaces. TechRxiv. Preprint. https://doi.org/10.36227/techrxiv.13636898.v3

$\mathrm{DOI}$

10.36227/techrxiv.13636898.v3 


\title{
Application of cylindrical IE-GSTC to physical metasurfaces
}

\author{
Srikumar Sandeep, Member, IEEE Albin Gasiewski, Fellow, IEEE Shao Ying Huang, Member, IEEE \\ and Andrew F Peterson, Fellow, IEEE
}

\begin{abstract}
The cylindrical IE-GSTC is validated by applying it to physical metasurfaces, i.e. metasurfaces defined by material properties and dimensions rather than by susceptibility tensor components. The previously reported IE-GSTC, which was formulated for zero thickness GSTC discontinuities, is extended to handle the finite thickness of physical metasurfaces. A simple analytical approach is used to extract the bianisotropic susceptibility tensor of concentric, multilayered, magneto-dielectric shells. Plane wave scattering by a physical metasurface constructed of four segments of multilayered, magneto-dielectric metasurface scatterers is used as an example problem to validate cylindrical IE-GSTC. A second example considers an opening on the cylindrical metasurface, confirming IE-GSTC can handle metasurfaces with openings. Third example is that of plane wave scattering by an eight segment metasurface. Good agreement is obtained between IE-GSTC results and full wave simulation results for all the cases. The paper concludes with a novel technique to handle PEC segments within a cylindrical metasurface.
\end{abstract}

Index Terms-GSTC, Integral equation, MoM, Cylindrical, Metasurface, Boundary condition, Susceptibility, Electromagnetic discontinuity, COMSOL

\section{INTRODUCTION}

$\mathbf{M}$ ETASURFACES, the 2D version of volumetric metamaterials, have provided unprecedented ability in the control and manipulation of electromagnetic waves. In comparison to metamaterials, metasurfaces are easier to fabricate. Metasurfaces are electrically thin surfaces constructed using subwavelength particles called metaatoms. By using engineered particles, metasurfaces can transform the phase, amplitude, polarization and propagation direction of the incident wave. Several review articles and books exist on the design, modeling and applications of planar metasurfaces [1]-[4]. Due to its deeply subwavelength thickness, physical metasurfaces can be abstracted as an infinitesimally thin layer of electric and magnetic surface polarization densities. Metasurfaces achieve their functionality by creating a spatial electromagnetic discontinuity. Mathematically, the discontinuity can be expressed by Generalized Sheet Transition Conditions (GSTCs) which relate the electric and magnetic field discontinuities to the electric and magnetic surface polarization densities [5], [6].

S. Sandeep is currently with the Department of Electronic Systems, NTNU, Trondheim, Norway. e-mail: sandeepsrikumar2013@gmail.com

A. J. Gasiewski is with the Department of Electrical and Computer Engineering, Unversity of Colorado, Boulder, CO, 80309 USA.

S. Y. Huang is with the Singapore University of Technology and Design (SUTD), Singapore.

A. F. Peterson is with the School of Electrical and Computer Engineering, Georgia Institute of Technology, Atlanta, GA, 30332 USA. e-mail: peterson@ece.gatech.edu
The surface polarization densities are related to the average of the fields on either side of the GSTC surface through the bianisotropic susceptibility tensor model [7]. It should be noted that even though physical metasurfaces have a finite subwavelength thickness, GSTCs which assume zero metasurface thickness are a very good approximation to such physical metasurfaces, i.e. the scattered field due to the physical metasurface approximates the field scattered by GSTC interface conditions.

GSTCs in combination with the bianisotropic susceptibility tensor model can be effectively used to simulate thin electromagnetic surfaces. Previous works in this approach include the application of a Finite Difference Frequency Domain (FDFD) method [8] and a Finite Element Method (FEM) [9] to the modeling of planar GSTCs. Even though most of the metasurfaces reported to date are planar, other canonical shapes such as cylindrical metasurfaces [10]-[13] and spherical metasurfaces [14], [15] are now being studied. Applications of conformal metasurfaces include illusion transformation [13], cloaking [12], high-gain antennas [16] and so on. GSTC bianisotropic susceptibility tensor model had been previously used for conformal metasurfaces. An Integral Equation - Method of Moment (IE-MoM) approach was applied to circular cylindrical metasurfaces in [10]. This was extended to cylindrical metasurfaces of arbitrary cross-section in [11]. Conformal transformation was used to synthesize surface electromagnetic tensor quantities for noncircular cylindrical metasurface in [17]. In [18], a digital signal processing based method is outlined for analysis and synthesis of cylindrical, bianisotropic metasurfaces. In [19], an inverse source technique was used to synthesize surface susceptibility tensor components of metasurfaces of arbitrary shape. The work addressed metasurfaces capable of generating radiation patterns with specified half-power beamwidth, nulls, polarization etc. Recently, asymptotic methods (GO, GTD) were applied to electrically large metasurfaces [20]. Even though mathematically rigorous, none of these works [10][12], [14], [19]-[23] have used physical metasurfaces.

One of the goals of this paper is to move from the bianisotropic susceptibility tensor based conformal GSTC surface models used in [10]-[12], [14], [19]-[23] to physical metasurfaces formed by curved metasurface scattering particles. In this work, validity of the cylindrical GSTC [10] is confirmed by comparing the scattering due to a cylindrical, physical metasurface to scattering by a corresponding GSTC contour. Scattering by a cylindrical GSTC contour was obtained by a slightly modified version of IE-GSTC formulation in [10]. 
The modification incorporates the finite thickness of physical metasurfaces. This modification is important to get accurate results for IE-GSTCs [10], [11], [22] when compared to physical metasurface scattering.

The organization of the paper is as follows. Section II extends the IE-MoM in [10] to handle a physical metasurface with non-zero thickness. The contents of this section closely follow [10]. Only the significant differences are explained. This is followed in section III by susceptibility tensor extraction of cylindrical, concentric, magneto-dielectric shell using an analytical approach. Section IV provides two examples. The metasurfaces in the two examples are constructed using metasurface particles which are part of the shell structure in section III. The second example has an opening which can be modeled using the IE-GSTC formulation, but with reduced accuracy. The IE-GSTC, using susceptibility tensors extracted using the method in section III, is used to simulate the scattering problem, with the results compared with a full wave simulation of the physical metasurface. The final section provides a detailed formulation for handling PEC segments in cylindrical metasurfaces with one numerical validation example.

\section{IE-MOM FOR FINITE THICKNESS CYLINDRICAL METASURFACE}

The IE-MoM described in [10] was limited to a cylindrical GSTC contour of zero thickness whose surface polarization densities are related to electric and magnetic fields on either side of the metasurface by the bianisotropic susceptibility tensor model. That formulation needs to be slightly extended to include the non-zero thickness of a physical metasurface. Instead of using a single contour for calculating MoM coefficients and integration of surface fields, two contours (one external and other internal to the metasurface) are used. This is shown in Fig. 1. Throughout this paper, there is no variation of material properties or excitation along the $z$ axis. The inner contour is $\Gamma_{2}$ and the outer contour is $\Gamma_{1}$, separated by the metasurface thickness $\delta$.

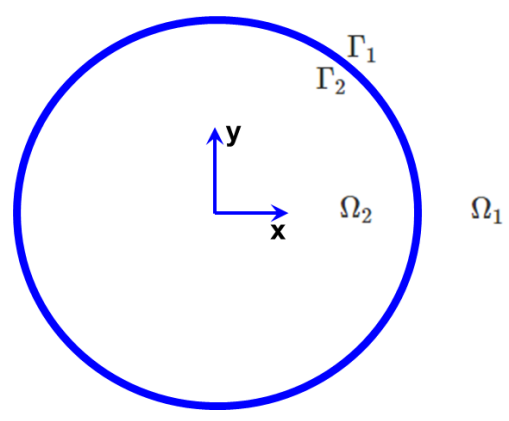

Fig. 1. Finite thickness cylindical metasurface

In this section, the extension of the formulation in [10] is outlined. A clear understanding of [10] is required to understand this section. The variables and notations follow
[10]. The 2D IEs for TM polarization given in equation (6) of [10] are modified to

$$
\begin{gathered}
E_{z 1}^{\mathrm{inc}}(\bar{\rho})+\oint_{\Gamma_{1}}\left[E_{z 1}\left(\bar{\rho}_{1}^{\prime}\right) \frac{\partial G_{0}\left(\bar{\rho} ; \bar{\rho}_{1}^{\prime}\right)}{\partial n^{\prime}}-\right. \\
\left.j \omega \mu_{0} H_{\phi 1}\left(\bar{\rho}_{1}^{\prime}\right) G_{0}\left(\bar{\rho} ; \bar{\rho}_{1}^{\prime}\right)\right] d \Gamma_{1}^{\prime}= \\
= \begin{cases}E_{z 1}(\bar{\rho}) & ; \bar{\rho} \in \Omega_{1} \\
0.5 E_{z 1}(\bar{\rho}) & ; \bar{\rho} \in \Gamma_{1} \\
0 & ; \bar{\rho} \in \Omega_{2}\end{cases} \\
E_{z 2}^{\mathrm{inc}}(\bar{\rho})-\oint_{\Gamma_{2}}\left[E_{z 2}\left(\bar{\rho}_{2}^{\prime}\right) \frac{\partial G_{0}\left(\bar{\rho} ; \bar{\rho}_{2}^{\prime}\right)}{\partial n^{\prime}}-\right. \\
\left.j \omega \mu_{0} H_{\phi 2}\left(\bar{\rho}_{2}^{\prime}\right) G_{0}\left(\bar{\rho} ; \bar{\rho}_{2}^{\prime}\right)\right] d \Gamma_{2}^{\prime}= \\
= \begin{cases}0 \quad & \\
0.5 E_{z 2}(\bar{\rho}) & ; \bar{\rho} \in \Gamma_{2} \\
E_{z 2}(\bar{\rho}) & ; \bar{\rho} \in \Omega_{2}\end{cases}
\end{gathered}
$$

where $\bar{\rho}_{1}^{\prime}$ and $\bar{\rho}_{2}^{\prime}$ denote contour integration position variables along $\Gamma_{1}$ and $\Gamma_{2}$ respectively. Such a distinction was not made in [10]. The subscripts 1,2 on the field variables denote the fields on domain $\Omega_{1}, \Omega_{2}$ respectively. $G_{0}\left(\rho ; \rho_{1}^{\prime}\right)=$ $-0.25 j H_{0}^{2}\left(k_{0}\left|\bar{\rho}-\bar{\rho}_{1}^{\prime}\right|\right)$ is the $2 \mathrm{D}$ Green's function. Derivative with respect to $n$ denotes directional derivative along the radial direction (pointing outwards). The 2D IEs for TE polarization given in equation (7) of [10] are modified to

$$
\begin{array}{r}
H_{z 1}^{\mathrm{inc}}(\bar{\rho})+\oint_{\Gamma_{1}}\left[H_{z 1}\left(\bar{\rho}_{1}^{\prime}\right) \frac{\partial G_{0}\left(\bar{\rho} ; \bar{\rho}_{1}^{\prime}\right)}{\partial n^{\prime}}+\right. \\
\left.j \omega \epsilon_{0} E_{\phi 1}\left(\bar{\rho}_{1}^{\prime}\right) G_{0}\left(\bar{\rho} ; \bar{\rho}_{1}^{\prime}\right)\right] d \Gamma_{1}^{\prime}= \\
= \begin{cases}H_{z 1}(\bar{\rho}) & ; \bar{\rho} \in \Omega_{1} \\
0.5 H_{z 1}(\bar{\rho}) & ; \bar{\rho} \in \Gamma_{1} \\
0 & ; \bar{\rho} \in \Omega_{2}\end{cases} \\
H_{z 2}^{\mathrm{inc}}(\bar{\rho}) \begin{array}{ll}
\oint_{\Gamma_{2}}\left[H_{z 2}\left(\bar{\rho}_{2}^{\prime}\right) \frac{\partial G_{0}\left(\bar{\rho} ; \bar{\rho}_{2}^{\prime}\right)}{\partial n^{\prime}}+\right. \\
\left.j \omega \epsilon_{0} E_{\phi 2}\left(\bar{\rho}_{2}^{\prime}\right) G_{0}\left(\bar{\rho} ; \bar{\rho}_{2}^{\prime}\right)\right] d \Gamma_{2}^{\prime}
\end{array} \\
= \begin{cases}0 \quad & \bar{\rho} \in \Omega_{1} \\
0.5 H_{z 2}(\bar{\rho}) & ; \bar{\rho} \in \Gamma_{2} \\
H_{z 2}(\bar{\rho}) & ; \bar{\rho} \in \Omega_{2}\end{cases}
\end{array}
$$

Following the same procedure as in [10], four integral equations are obtained using (1), (2) and circular metasurface synthesis equations given in equation (4) of [10]. These four equations are used to solve for the four tangential fields $E_{z 1}\left(\bar{\rho}_{1}^{\prime}\right), H_{\phi 1}\left(\bar{\rho}_{1}^{\prime}\right), E_{\phi 1}\left(\bar{\rho}_{1}^{\prime}\right)$ and $H_{z 1}\left(\bar{\rho}_{1}^{\prime}\right)$ on the exterior contour $\Gamma_{1}$. The equations are given as

$$
\begin{gathered}
\frac{E_{z 1}\left(\bar{\rho}_{1}\right)}{2}-\oint_{\Gamma_{1}}\left[E_{z 1}\left(\bar{\rho}_{1}^{\prime}\right) \frac{\partial G_{0}\left(\bar{\rho}_{1} ; \bar{\rho}_{1}^{\prime}\right)}{\partial n^{\prime}}-j \omega \mu_{0}\right. \\
\left.H_{\phi 1}\left(\bar{\rho}_{1}^{\prime}\right) G_{0}\left(\bar{\rho}_{1} ; \bar{\rho}_{1}^{\prime}\right)\right] d \Gamma_{1}^{\prime}=E_{z 1}^{\mathrm{inc}}\left(\bar{\rho}_{1}\right) ; \bar{\rho}_{1} \in \Gamma_{1}
\end{gathered}
$$




$$
\begin{aligned}
& \oint_{\Gamma_{2}}\left[A_{11}\left(\bar{\rho}_{c}^{\prime}\right) E_{z 1}\left(\bar{\rho}_{1}^{\prime}\right)+A_{12}\left(\bar{\rho}_{c}^{\prime}\right) H_{\phi 1}\left(\bar{\rho}_{1}^{\prime}\right)+A_{13}\left(\bar{\rho}_{c}^{\prime}\right) E_{\phi 1}\left(\bar{\rho}_{1}^{\prime}\right)\right. \\
& \left.+A_{14}\left(\bar{\rho}_{c}^{\prime}\right) H_{z 1}\left(\bar{\rho}_{1}^{\prime}\right)\right] \frac{\partial G_{0}\left(\bar{\rho}_{2} ; \bar{\rho}_{2}^{\prime}\right)}{\partial n^{\prime}}-j \omega \mu_{0}\left[A_{21}\left(\bar{\rho}_{c}^{\prime}\right) E_{z 1}\left(\bar{\rho}_{1}^{\prime}\right)+\right. \\
& \left.A_{22}\left(\bar{\rho}_{c}^{\prime}\right) H_{\phi 1}\left(\bar{\rho}_{1}^{\prime}\right)+A_{23}\left(\bar{\rho}_{c}^{\prime}\right) E_{\phi 1}\left(\bar{\rho}_{1}^{\prime}\right)+A_{24}\left(\bar{\rho}_{c}^{\prime}\right) H_{z 1}\left(\bar{\rho}_{1}^{\prime}\right)\right] \\
& G_{0}\left(\bar{\rho}_{2} ; \bar{\rho}_{2}^{\prime}\right) d \Gamma_{2}^{\prime}+0.5\left[A_{11}\left(\bar{\rho}_{c}\right) E_{z 1}\left(\bar{\rho}_{1}\right)+A_{12}\left(\bar{\rho}_{c}\right) H_{\phi 1}\left(\bar{\rho}_{1}\right)+\right. \\
& \left.A_{13}\left(\bar{\rho}_{c}\right) E_{\phi 1}\left(\bar{\rho}_{1}\right)+A_{14}\left(\bar{\rho}_{c}\right) H_{z 1}\left(\bar{\rho}_{1}\right)\right]=E_{z 2}^{\mathrm{inc}}\left(\bar{\rho}_{2}\right) ; \bar{\rho}_{2} \in \Gamma_{2} \\
& \frac{H_{z 1}\left(\bar{\rho}_{1}\right)}{2}-\oint_{\Gamma_{1}}\left[H_{z 1}\left(\bar{\rho}_{1}^{\prime}\right) \frac{\partial G_{0}\left(\bar{\rho}_{1} ; \bar{\rho}_{1}^{\prime}\right)}{\partial n^{\prime}}+j \omega \epsilon_{0}\right. \\
& \left.E_{\phi 1}\left(\bar{\rho}_{1}^{\prime}\right) G_{0}\left(\bar{\rho}_{1} ; \bar{\rho}_{1}^{\prime}\right)\right] d \Gamma_{1}^{\prime}=H_{z 1}^{\mathrm{inc}}\left(\bar{\rho}_{1}\right) ; \bar{\rho}_{1} \in \Gamma_{1} \\
& \oint_{\Gamma_{2}}\left[A_{41}\left(\bar{\rho}_{c}^{\prime}\right) E_{z 1}\left(\bar{\rho}_{1}^{\prime}\right)+A_{42}\left(\bar{\rho}_{c}^{\prime}\right) H_{\phi 1}\left(\bar{\rho}_{1}^{\prime}\right)+A_{43}\left(\bar{\rho}_{c}^{\prime}\right) E_{\phi 1}\left(\bar{\rho}_{1}^{\prime}\right)\right. \\
& \left.+A_{44}\left(\bar{\rho}_{c}^{\prime}\right) H_{z 1}\left(\bar{\rho}_{1}^{\prime}\right)\right] \frac{\partial G_{0}\left(\bar{\rho}_{2} ; \bar{\rho}_{2}^{\prime}\right)}{\partial n^{\prime}}+j \omega \epsilon_{0}\left[A_{31}\left(\bar{\rho}_{c}^{\prime}\right) E_{z 1}\left(\bar{\rho}_{1}^{\prime}\right)+\right. \\
& \left.A_{32}\left(\bar{\rho}_{c}^{\prime}\right) H_{\phi 1}\left(\bar{\rho}_{1}^{\prime}\right)+A_{33}\left(\bar{\rho}_{c}^{\prime}\right) E_{\phi 1}\left(\bar{\rho}_{1}^{\prime}\right)+A_{34}\left(\bar{\rho}_{c}^{\prime}\right) H_{z 1}\left(\bar{\rho}_{1}^{\prime}\right)\right] \\
& G_{0}\left(\bar{\rho}_{2} ; \bar{\rho}_{2}^{\prime}\right) d \Gamma_{2}^{\prime}+0.5\left[A_{41}\left(\bar{\rho}_{c}\right) E_{z 1}\left(\bar{\rho}_{1}\right)+A_{42}\left(\bar{\rho}_{c}\right) H_{\phi 1}\left(\bar{\rho}_{1}\right)+\right. \\
& \left.A_{43}\left(\bar{\rho}_{c}\right) E_{\phi 1}\left(\bar{\rho}_{1}\right)+A_{44}\left(\bar{\rho}_{c}\right) H_{z 1}\left(\bar{\rho}_{1}\right)\right]=H_{z 2}^{\mathrm{inc}}\left(\bar{\rho}_{2}\right) ; \bar{\rho}_{2} \in \Gamma_{2}
\end{aligned}
$$

where the $A_{i j}\left(\bar{\rho}_{c}^{\prime}\right)$ are elements of the $4 \times 4$ matrix $\mathbf{A}\left(\bar{\rho}_{c}^{\prime}\right)$ [10]. This matrix, which is a function of bianisotropic susceptibility tensor components (which vary azimuthally), relates the tangential electric and magnetic field components on the inner contour and the outer contour. $\bar{\rho}_{c}^{\prime}$ denotes the center of the metasurface, i.e. $\bar{\rho}_{c}^{\prime}=\bar{\rho}_{1}^{\prime}-0.5 \delta \hat{\rho}$. Since the material properties of the metasurface vary azimuthally, the bianisotropic susceptibility tensor components and matrix $\mathbf{A}\left(\bar{\rho}_{c}^{\prime}\right)$ also varies with position. To be precise the matrix $\mathbf{A}\left(\bar{\rho}_{c}^{\prime}\right)$ is defined as

$$
\begin{aligned}
\mathbf{F}_{\mathbf{2}}\left(\bar{\rho}_{\mathbf{2}}^{\prime}\right) & =\mathbf{A}\left(\bar{\rho}_{\mathbf{c}}^{\prime}\right) \mathbf{F}_{\mathbf{1}}\left(\bar{\rho}_{\mathbf{1}}^{\prime}\right) \\
\mathbf{A}\left(\bar{\rho}_{\mathbf{c}}^{\prime}\right) & =\mathbf{A}_{\mathbf{2}}^{-\mathbf{1}}\left(\bar{\rho}_{\mathbf{c}}^{\prime}\right) \mathbf{A}_{\mathbf{1}}\left(\bar{\rho}_{\mathbf{c}}^{\prime}\right)
\end{aligned}
$$

where

$$
\begin{aligned}
& \mathbf{F}_{\mathbf{2}}\left(\bar{\rho}_{\mathbf{2}}^{\prime}\right)=\left[E_{z 2}\left(\bar{\rho}_{2}^{\prime}\right) H_{\phi 2}\left(\bar{\rho}_{2}^{\prime}\right) E_{\phi 2}\left(\bar{\rho}_{2}^{\prime}\right) H_{z 2}\left(\bar{\rho}_{2}^{\prime}\right)\right]^{T} \\
& \mathbf{F}_{\mathbf{1}}\left(\bar{\rho}_{\mathbf{1}}^{\prime}\right)=\left[E_{z 1}\left(\bar{\rho}_{1}^{\prime}\right) H_{\phi 1}\left(\bar{\rho}_{1}^{\prime}\right) E_{\phi 1}\left(\bar{\rho}_{1}^{\prime}\right) H_{z 1}\left(\bar{\rho}_{1}^{\prime}\right)\right]^{T}
\end{aligned}
$$

The matrices $\mathbf{A}_{1}\left(\bar{\rho}_{c}^{\prime}\right)$ and $\mathbf{A}_{2}\left(\bar{\rho}_{c}^{\prime}\right)$, which are defined in equations (22), (23) in [10], are repeated in equations (6) and (7) for completeness.

The integration contours in (3a) - (3d) should be carefully noted. They are different from the corresponding equations in [10]. Similar to [10], the IEs (3a) - (3d) are converted to an MoM system of linear equations using pulse basis functions and point matching. The MoM system of linear equations reads

$$
\left[\begin{array}{llll}
\mathrm{Z}_{11} & \mathrm{Z}_{12} & \mathrm{Z}_{13} & \mathrm{Z}_{14} \\
\mathrm{Z}_{21} & \mathrm{Z}_{22} & \mathrm{Z}_{23} & \mathrm{Z}_{24} \\
\mathbf{Z}_{31} & \mathrm{Z}_{32} & \mathrm{Z}_{33} & \mathrm{Z}_{34} \\
\mathrm{Z}_{41} & \mathrm{Z}_{42} & \mathrm{Z}_{43} & \mathrm{Z}_{44}
\end{array}\right]\left[\begin{array}{l}
\mathrm{x}_{1} \\
\mathrm{x}_{2} \\
\mathrm{x}_{3} \\
\mathrm{x}_{4}
\end{array}\right]=\left[\begin{array}{l}
\mathrm{b}_{1} \\
\mathrm{~b}_{2} \\
\mathrm{~b}_{3} \\
\mathrm{~b}_{4}
\end{array}\right]
$$

where the unknown vectors are

$$
\begin{aligned}
& \mathbf{x}_{\mathbf{1}}=\left[E_{z 1,1}, \cdots, E_{z 1, \mathrm{~N}}\right]^{T} \\
& \mathbf{x}_{\mathbf{2}}=\left[H_{\phi 1,1}, \cdots, H_{\phi 1, \mathrm{~N}}\right]^{T} \\
& \mathbf{x}_{\mathbf{3}}=\left[E_{\phi 1,1}, \cdots, E_{\phi 1, \mathrm{~N}}\right]^{T} \\
& \mathbf{x}_{\mathbf{4}}=\left[H_{z 1,1}, \cdots, H_{z 1, \mathrm{~N}}\right]^{T}
\end{aligned}
$$

$\mathrm{N}$ is the total number of basis functions. The $\mathbf{Z}$ matrix coefficients and the excitation vector are as follows

$$
\begin{aligned}
& Z_{11, \mathrm{mn}}=p_{\mathrm{mn}}^{1} \\
& Z_{12, \mathrm{mn}}=r_{\mathrm{mn}}^{1} \\
& Z_{13, \mathrm{mn}}=0 \\
& Z_{14, \mathrm{mn}}=0
\end{aligned}
$$

$$
\begin{aligned}
& Z_{21, \mathrm{mn}}=A_{11, \mathrm{n}} q_{\mathrm{mn}}^{2}-A_{21, \mathrm{n}} r_{\mathrm{mn}}^{2} \\
& Z_{22, \mathrm{mn}}=A_{12, \mathrm{n}} q_{\mathrm{mn}}^{2}-A_{22, \mathrm{n}} r_{\mathrm{mn}}^{2} \\
& Z_{23, \mathrm{mn}}=A_{13, \mathrm{n}} q_{\mathrm{mn}}^{2}-A_{23, \mathrm{n}} r_{\mathrm{mn}}^{2} \\
& Z_{24, \mathrm{mn}}=A_{14, \mathrm{n}} q_{\mathrm{mn}}^{2}-A_{24, \mathrm{n}} r_{\mathrm{mn}}^{2}
\end{aligned}
$$

$$
\begin{aligned}
& Z_{31, \mathrm{mn}}=0 \\
& Z_{32, \mathrm{mn}}=0 \\
& Z_{33, \mathrm{mn}}=-s_{\mathrm{mn}}^{1} \\
& Z_{34, \mathrm{mn}}=p_{\mathrm{mn}}^{1}
\end{aligned}
$$

$$
\begin{aligned}
& Z_{41, \mathrm{mn}}=A_{41, \mathrm{n}} q_{\mathrm{mn}}^{2}+A_{31, \mathrm{n}} s_{\mathrm{mn}}^{2} \\
& Z_{42, \mathrm{mn}}=A_{42, \mathrm{n}} q_{\mathrm{mn}}^{2}+A_{32, \mathrm{n}} s_{\mathrm{mn}}^{2} \\
& Z_{43, \mathrm{mn}}=A_{43, \mathrm{n}} q_{\mathrm{mn}}^{2}+A_{33, \mathrm{n}} s_{\mathrm{mn}}^{2} \\
& Z_{44, \mathrm{mn}}=A_{44, \mathrm{n}} q_{\mathrm{mn}}^{2}+A_{34, \mathrm{n}} s_{\mathrm{mn}}^{2}
\end{aligned}
$$

where $A_{\mathrm{ij}, n}$ is the value of matrix elements $A_{\mathrm{ij}}$ at the $n^{\text {th }}$ discretization segment. It is these matrix elements which connect IE-GSTC to susceptibility tensor components. The coefficients $p_{\mathrm{mn}}^{1}, q_{\mathrm{mn}}^{2}, r_{\mathrm{mn}}^{1}, r_{\mathrm{mn}}^{2}, s_{\mathrm{mn}}^{1}, s_{\mathrm{mn}}^{2}$ are calculated as

$$
\begin{aligned}
& p_{\mathrm{mn}}^{1}=\frac{1}{2} \delta_{\mathrm{mn}}-\int_{s_{n 1}} \frac{\partial G_{0}\left(\bar{\rho}_{m 1} ; \bar{\rho}_{1}^{\prime}\right)}{\partial n^{\prime}} d \Gamma_{1}^{\prime} \\
& q_{\mathrm{mn}}^{2}=\frac{1}{2} \delta_{\mathrm{mn}}+\int_{s_{n 2}} \frac{\partial G_{0}\left(\bar{\rho}_{m 2} ; \bar{\rho}_{2}^{\prime}\right)}{\partial n^{\prime}} d \Gamma_{2}^{\prime} \\
& r_{\mathrm{mn}}^{1}=j \omega \mu_{0} \int_{s_{n 1}} G_{0}\left(\bar{\rho}_{m 1} ; \bar{\rho}_{1}^{\prime}\right) d \Gamma_{1}^{\prime} \\
& r_{\mathrm{mn}}^{2}=j \omega \mu_{0} \int_{s_{n 2}} G_{0}\left(\bar{\rho}_{m 2} ; \bar{\rho}_{2}^{\prime}\right) d \Gamma_{2}^{\prime} \\
& s_{\mathrm{mn}}^{1}=j \omega \epsilon_{0} \int_{s_{n 1}} G_{0}\left(\bar{\rho}_{m 1} ; \bar{\rho}_{1}^{\prime}\right) d \Gamma_{1}^{\prime} \\
& s_{\mathrm{mn}}^{2}=j \omega \epsilon_{0} \int_{s_{n 2}} G_{0}\left(\bar{\rho}_{m 2} ; \bar{\rho}_{1}^{\prime}\right) d \Gamma_{2}^{\prime}
\end{aligned}
$$

$s_{n 1}, s_{n 2}$ denote the $n^{\text {th }}$ discretization segments on contours $\Gamma_{1}, \Gamma_{2}$, respectively. $\bar{\rho}_{m 1}, \bar{\rho}_{m 2}$ are the middle points of the $m^{\text {th }}$ segments on contours $\Gamma_{1}, \Gamma_{2} . \delta_{m n}$ is the Kronecker delta function. The superscript 1 or 2 in $p_{\mathrm{mn}}^{1}, q_{\mathrm{mn}}^{2}, r_{\mathrm{mn}}^{1}, r_{\mathrm{mn}}^{2}, s_{\mathrm{mn}}^{1}, s_{\mathrm{mn}}^{2}$ denotes whether the integration is performed along the outer 


$$
\begin{aligned}
& \mathbf{A}_{\mathbf{1}}\left(\bar{\rho}_{\mathbf{c}}^{\prime}\right)= {\left[\begin{array}{cccc}
\frac{j \omega \epsilon_{0} \chi_{\mathrm{ee}}^{\phi z}\left(\bar{\rho}_{c}^{\prime}\right)}{2} & \frac{j \omega c_{0}^{-1} \chi_{\mathrm{em}}^{\phi \phi}\left(\bar{\rho}_{c}^{\prime}\right)}{2} & \frac{j \omega \epsilon_{0} \chi_{\mathrm{ee}}^{\phi \phi}\left(\bar{\rho}_{c}^{\prime}\right)}{2} & \frac{2+j \omega c_{0}^{-1} \chi_{\mathrm{em}}^{\phi z}\left(\bar{\rho}_{c}^{\prime}\right)}{2} \\
\frac{j \omega \epsilon_{0} \chi_{\mathrm{e}}^{z z}\left(\bar{\rho}_{c}^{\prime}\right)}{2} & \frac{-2+j \omega c_{0}^{-1} \chi_{\mathrm{em}}^{z \phi}\left(\bar{\rho}_{c}^{\prime}\right)}{2} & \frac{j \omega \epsilon_{0} \chi_{\mathrm{ee}}^{z \phi}\left(\bar{\rho}_{c}^{\prime}\right)}{2} & \frac{j \omega c_{0}^{-1} \chi_{\mathrm{em}}^{z z}\left(\bar{\rho}_{c}^{\prime}\right)}{2} \\
\frac{-2+j \omega c_{0}^{-1} \chi_{\mathrm{me}}^{\phi z}\left(\bar{\rho}_{c}^{\prime}\right)}{2} & \frac{j \omega \mu_{0} \chi_{\mathrm{mm}}^{\phi \phi}\left(\bar{\rho}_{c}^{\prime}\right)}{2} & \frac{j \omega c_{0}^{-1} \chi_{\mathrm{me}}^{\phi \phi}\left(\bar{\rho}_{c}^{\prime}\right)}{2} & \frac{j \omega \mu_{0} \chi_{\mathrm{mm}}^{\phi z}\left(\bar{\rho}_{c}^{\prime}\right)}{2} \\
\frac{j \omega c_{0}^{-1} \chi_{\mathrm{me}}^{z z}\left(\bar{\rho}_{c}^{\prime}\right)}{2} & \frac{j \omega \mu_{0} \chi_{\mathrm{mm}}^{z \phi}\left(\bar{\rho}_{c}^{\prime}\right)}{2} & \frac{2+j \omega c_{0}^{-1} \chi_{\mathrm{me}}^{z \phi}\left(\bar{\rho}_{c}^{\prime}\right)}{2} & \frac{j \omega \mu_{0} \chi_{\mathrm{mm}}^{z z}\left(\bar{\rho}_{c}^{\prime}\right)}{2}
\end{array}\right] } \\
& \mathbf{A}_{\mathbf{2}}\left(\bar{\rho}_{\mathbf{c}}^{\prime}\right)=\left[\begin{array}{cccc}
\frac{j \omega \epsilon_{0} \chi_{\mathrm{ee}}^{\phi z}\left(\bar{\rho}_{c}^{\prime}\right)}{-2} & \frac{j \omega c_{0}^{-1} \chi_{\mathrm{em}}^{\phi \phi}\left(\bar{\rho}_{c}^{\prime}\right)}{-2} & \frac{j \omega \epsilon_{0} \chi_{\mathrm{ee}}^{\phi \phi}\left(\bar{\rho}_{c}^{\prime}\right)}{-2} & \frac{2-j \omega c_{0}^{-1} \chi_{\mathrm{em}}^{\phi z}\left(\bar{\rho}_{c}^{\prime}\right)}{2} \\
\frac{j \omega \epsilon_{0} \chi_{\mathrm{ee}}^{z z}\left(\bar{\rho}_{c}^{\prime}\right)}{-2} & \frac{-2-j \omega c_{0}^{-1} \chi_{\mathrm{em}}^{z \phi}\left(\bar{\rho}_{c}^{\prime}\right)}{2} & \frac{j \omega \epsilon_{0} \chi_{\mathrm{ee}}^{z \phi}\left(\bar{\rho}_{c}^{\prime}\right)}{-2} & \frac{j \omega c_{0}^{-1} \chi_{\mathrm{em}}^{z z}\left(\bar{\rho}_{c}^{\prime}\right)}{-2} \\
\frac{-2-j \omega c_{0}^{-1} \chi_{\mathrm{me}}^{\phi z}\left(\bar{\rho}_{c}^{\prime}\right)}{2} & \frac{j \omega \mu_{0} \chi_{\mathrm{mm}}^{\phi \phi}\left(\bar{\rho}_{c}^{\prime}\right)}{-2} & \frac{j \omega c_{0}^{-1} \chi_{\mathrm{me}}^{\phi \phi}\left(\bar{\rho}_{c}^{\prime}\right)}{-2} & \frac{j \omega \mu_{0} \chi_{\mathrm{mm}}^{\phi z}\left(\bar{\rho}_{c}^{\prime}\right)}{-2} \\
\frac{j \omega c_{0}^{-1} \chi_{\mathrm{me}}^{z z}\left(\bar{\rho}_{c}^{\prime}\right)}{-2} & \frac{j \omega \mu_{0} \chi_{\mathrm{mm}}^{z \phi}\left(\bar{\rho}_{c}^{\prime}\right)}{-2} & \frac{2-j \omega c_{0}^{-1} \chi_{\mathrm{me}}^{z p}\left(\bar{\rho}_{c}^{\prime}\right)}{2} & \frac{j \omega \mu_{0} \chi_{\mathrm{mm}}^{z z}\left(\bar{\rho}_{c}^{\prime}\right)}{-2}
\end{array}\right]
\end{aligned}
$$

or inner contour. The excitation vector components are given by

$$
\begin{gathered}
b_{1, m}=E_{z 1}^{\mathrm{inc}}\left(\bar{\rho}_{1, m}\right), b_{2, m}=E_{z 2}^{\mathrm{inc}}\left(\bar{\rho}_{2, m}\right) \\
b_{3, m}=H_{z 1}^{\mathrm{inc}}\left(\bar{\rho}_{1, m}\right), b_{4, m}=H_{z 2}^{\mathrm{inc}}\left(\bar{\rho}_{2, m}\right)
\end{gathered}
$$

These four excitation vectors provide four possibilites: $b_{1, m}$ is for a TM excitation external to the metasurface, $b_{2, m}$ is for a TM excitation internal to the metasurface, $b_{3, m}$ is for a TE excitation external to the metasurface, and $b_{4, m}$ is for a TE excitation internal to the metasurface.

\section{SUSCEPTIBILITY EXTRACTION OF CYLINDRICAL, MULTILAYERED MAGNETO-DIELECTRIC SHELL}

In this section, the bianisotropic susceptibility tensor components of a cylindrical, multilayered, magneto-dielectric shell are extracted. The geometry is shown in Fig. 2. Even though a three layered geometry is shown, the method can be used for any number of layers. The medium inside and outside the shell is vacuum. $\mathrm{TM}_{\mathrm{z}}$ polarization is assumed. Follow-

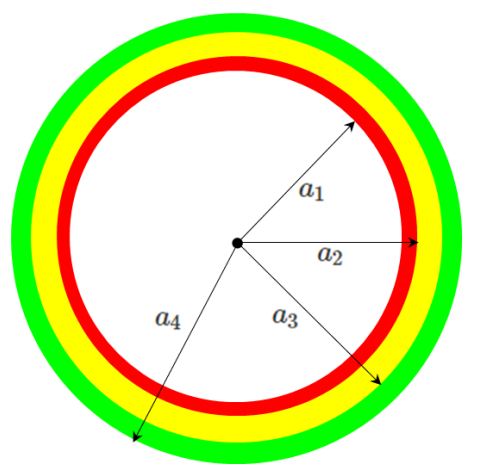

Fig. 2. Three layered cylindrical, magneto-dielectric shell

ing equation (4) in [10], the differential field components across the cylindrical metasurface $\Delta E_{z}, \Delta H_{\phi}, \Delta H_{z}, \Delta E_{\phi}$ are related to the average of fields across the metasurface $E_{z, \text { av }}, H_{\phi, \text { av }}, E_{\phi, \text { av }}, H_{z, \text { av }}$, through the following metasurface synthesis expressions:

$$
\begin{array}{r}
{\left[\begin{array}{c}
-\Delta H_{z} \\
\Delta H_{\phi}
\end{array}\right]=j \omega \epsilon_{0}\left[\begin{array}{cc}
\chi_{\mathrm{ee}}^{\phi \phi} & \chi_{\mathrm{ee}}^{\phi z} \\
\chi_{\mathrm{ee}}^{z \phi} & \chi_{\mathrm{ee}}^{z z}
\end{array}\right]\left[\begin{array}{c}
E_{\phi, \mathrm{av}} \\
E_{z, \mathrm{av}}
\end{array}\right]+j \omega \sqrt{\mu_{0} \epsilon_{0}}} \\
{\left[\begin{array}{cc}
\chi_{\mathrm{em}}^{\phi \phi} & \chi_{\mathrm{em}}^{\phi z} \\
\chi_{\mathrm{em}}^{z \phi} & \chi_{\mathrm{em}}^{z z}
\end{array}\right]\left[\begin{array}{c}
H_{\phi, \mathrm{av}} \\
H_{z, \mathrm{av}}
\end{array}\right]}
\end{array}
$$

$$
\begin{array}{r}
{\left[\begin{array}{c}
\Delta E_{z} \\
-\Delta E_{\phi}
\end{array}\right]=j \omega \sqrt{\mu_{0} \epsilon_{0}}\left[\begin{array}{cc}
\chi_{\mathrm{me}}^{\phi \phi} & \chi_{\mathrm{me}}^{\phi z} \\
\chi_{\mathrm{me}}^{z \phi} & \chi_{\mathrm{me}}^{z z}
\end{array}\right]\left[\begin{array}{c}
E_{\phi, \mathrm{av}} \\
E_{z, \mathrm{av}}
\end{array}\right]+j \omega \mu_{0}} \\
{\left[\begin{array}{cc}
\chi_{\mathrm{mm}}^{\phi \phi} & \chi_{\mathrm{mm}}^{\phi z} \\
\chi_{\mathrm{mm}}^{z \phi} & \chi_{\mathrm{mm}}^{z z}
\end{array}\right]\left[\begin{array}{c}
H_{\phi, \mathrm{av}} \\
H_{z, \mathrm{av}}
\end{array}\right]}
\end{array}
$$

For the case of $\mathrm{TM}_{\mathrm{z}}$ polarization, they reduce to

$$
\begin{gathered}
\Delta E_{z}=j \omega \sqrt{\mu_{0} \epsilon_{0}} \chi_{\mathrm{me}}^{\phi z} E_{z, \mathrm{av}}+j \omega \mu_{0} \chi_{\mathrm{mm}}^{\phi \phi} H_{\phi, \mathrm{av}} \\
\Delta H_{\phi}=j \omega \epsilon_{0} \chi_{\mathrm{ee}}^{z z} E_{z, \mathrm{av}}+j \omega \sqrt{\mu_{0} \epsilon_{0}} \chi_{\mathrm{em}}^{z \phi} H_{\phi, \mathrm{av}}
\end{gathered}
$$

Since there are four unknown susceptibility components in the above equation, two different excitation problems are solved to obtain the four susceptibility components. The two excitations used are shown in Fig. 3. The first problem is that of an infinite, electric line source at the origin and the second excitation problem is that of a cylindrical surface electric current density of radius $a_{s}$, where $a_{s}>a_{4}$. The two scattering problems are simple boundary value problems, which can be either solved analytically [24] or simulated using COMSOL. In the case of analytical solution, the vector

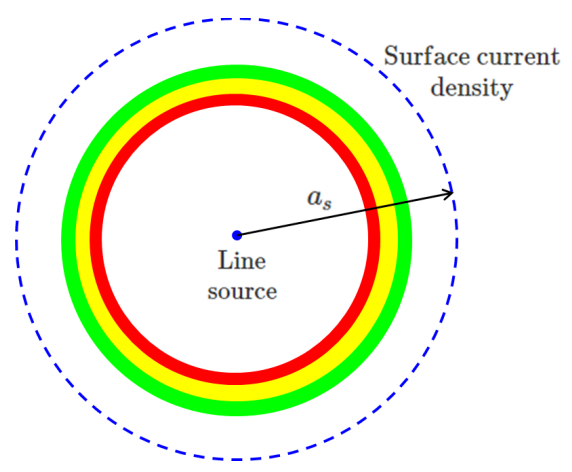

Fig. 3. Bianisotropic susceptibility tensor extraction using two sources: (1) Infinite electric line source at origin, $\bar{J}=\delta(x) \delta(y) \hat{z} \mathrm{Am}^{-2}$ shown as a blue dot (2) Cylindrical electric surface current density of radius $a_{s}, \bar{J}_{s}=$ $\delta\left(\rho-a_{s}\right) \hat{z} \mathrm{Am}^{-1}$ shown as a dashed, blue circle.

magnetic potential, electric field and magnetic field in each region can be expressed in terms of Bessel functions:

$$
\begin{array}{r}
A_{z, i}(\rho)=C_{i} J_{0}\left(k_{i} \rho\right)+D_{i} Y_{0}\left(k_{i} \rho\right) \\
E_{z, i}(\rho)=-j \omega\left[C_{i} J_{0}\left(k_{i} \rho\right)+D_{i} Y_{0}\left(k_{i} \rho\right)\right] \\
H_{\phi, i}(\rho)=\frac{k_{i}}{\mu_{i}}\left[C_{i} J_{1}\left(k_{i} \rho\right)+D_{i} Y_{1}\left(k_{i} \rho\right)\right]
\end{array}
$$


where $J_{0}(\cdot), Y_{0}(\cdot)$ are Bessel functions of the first and second kind, respectively. $k_{i}, \mu_{i}$ are the wavenumber and permeability of region $i$. For the case of a cylindrical surface current density excitation of the three layered, magneto-dielectric cylinder, the total number of regions is 6 (the surface current density discontinuity splits the external medium into two separate regions). Applying the continuity of electric and magnetic fields across the region boundaries and the magnetic field discontinuity caused by surface current density, the unknowns $C_{i}, D_{i}$ can be obtained. Details of this problem can be found in appendix A.

Once the two excitation problems are solved, the susceptibility tensor components are obtained as follows

$j \frac{\omega}{c_{0}}\left[\begin{array}{cccc}E_{z, \mathrm{av}}^{(1)} & \eta_{0} H_{\phi, \mathrm{av}}^{(1)} & 0 & 0 \\ 0 & 0 & \frac{E_{z, \mathrm{av}}^{(1)}}{\eta_{0}} & H_{\phi, \mathrm{av}}^{(1)} \\ E_{z, \mathrm{av}}^{(2)} & \eta_{0} H_{\phi, \mathrm{av}}^{(2)} & 0 & 0 \\ 0 & 0 & \frac{E_{z, \mathrm{av}}^{(2)}}{\eta_{0}} & H_{\phi, \mathrm{av}}^{(2)}\end{array}\right]\left[\begin{array}{c}\chi_{\mathrm{me}}^{\phi z} \\ \chi_{\mathrm{mm}}^{\phi \phi} \\ \chi_{\mathrm{ee}}^{z z} \\ \chi_{\mathrm{em}}^{z \phi}\end{array}\right]=\left[\begin{array}{c}\Delta E_{z}^{(1)} \\ \Delta H_{\phi}^{(1)} \\ \Delta E_{z}^{(2)} \\ \Delta H_{\phi}^{(2)}\end{array}\right]$

where the superscript (1) denotes line source excitation fields and (2) denotes the cylindrical surface current density excitation. It should be noted that the magneto-dielectric shells and excitations considered are azimuthally invariant, hence the resultant tangential fields and the extractated susceptibilities have no dependance along the $\phi$ direction.

\section{NUMERICAL VALIDATION}

This section presents examples of cylindrical metasurfaces which use IE-GSTC and the susceptibility extraction method described in the the previous sections. COMSOL software is used for full wave simulations.

\section{A. Four segment cylindrical metasurface excited by plane wave}

The cylindrical metasurface shown in Fig. 4 consists of four segments (quadrants). Each segment is a multilayered, concentric, magneto-dielectric shell. The susceptibility tensor components of each segment can be extracted using the method in the previous section. Referring to Fig. 4, different annular regions are identified as follows: region 1: $0 \leq \rho<a_{1}$, region 2: $a_{1} \leq \rho<a_{2}$, region 3: $a_{2} \leq \rho<a_{3}$, region 4: $a_{3} \leq \rho<a_{4}$ and region 5: $a_{4} \leq \rho<\infty$. The metasurface is surrounded by vacuum, i.e. region 1 and region 5 are vacuum. The material properties of each region (represented by subscript number) in the four quadrants are given in table I. The susceptibility tensors of the quadrants are also given. The dimensions are: $a_{1}=5 \lambda, a_{2}=5 \lambda+\frac{\lambda}{24}, a_{3}=a_{2}+\frac{\lambda}{24}$, $a_{4}=a_{3}+\frac{\lambda}{20}$. The susceptibility component extraction from the last section is performed four times to get the component values. In the IE-GSTC code, the matrix $A$ is varied depending on the position. For instance, for the first quadrant, $A$ is calculated using tensor components in the first row of table I.

The structure is excited by a plane wave $\bar{E}^{\text {inc }}(x)=$ $e^{-j k_{0} x} \hat{z}$. The total field along a line from $(0,0)$ to $(10 \lambda, 0)$ is plotted in Fig. 5 and Fig. 6. Good agreement between

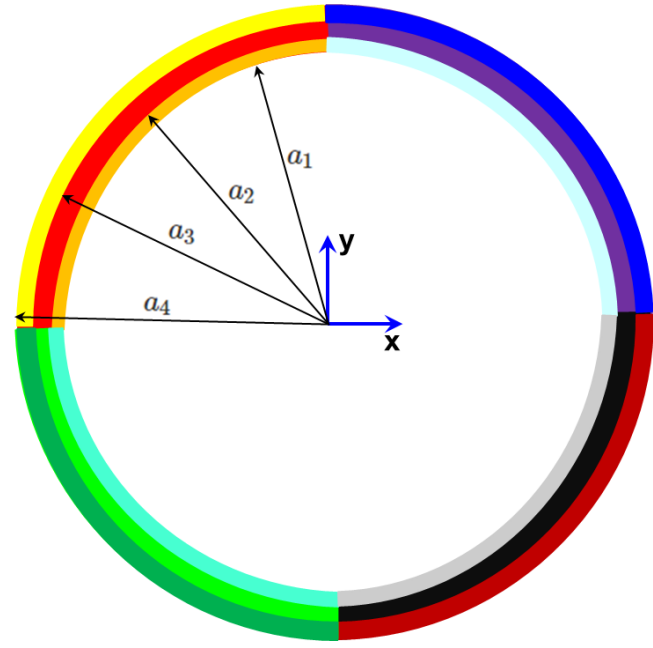

Fig. 4. Four segment cylindrical metasurface.

\begin{tabular}{|l|l|l|}
\hline Quadrant & $\begin{array}{l}\text { Material } \\
\text { properties }\end{array}$ & Susceptibility tensor \\
\hline & $\epsilon_{r 1}=1, \mu_{r 1}=1$ & $\chi_{\mathrm{me}}^{\phi z}=j 2.55236 \times 10^{-3}$ \\
Quadrant 1 & $\epsilon_{r 2}=9, \mu_{r 2}=2$ & $\chi_{\mathrm{mm}}^{\phi \phi}=6.08697 \times 10^{-2}$ \\
& $\epsilon_{r 3}=4, \mu_{r 3}=1$ & $\chi_{\mathrm{ez}}^{z z}=1.97682 \times 10^{-1}$ \\
& $\epsilon_{r 4}=9, \mu_{r 4}=2$ & $\chi_{\mathrm{em}}^{z \phi}=j 1.42107 \times 10^{-2}$ \\
& $\epsilon_{r 5}=1, \mu_{r 5}=1$ & \\
\hline & $\epsilon_{r 1}=1, \mu_{r 1}=1$ & $\chi_{\mathrm{me}}^{\phi z}=-j 1.79047 \times 10^{-5}$ \\
& $\epsilon_{r 2}=4, \mu_{r 2}=1$ & $\chi_{\mathrm{mm}}^{\phi \phi}=5.27727 \times 10^{-3}$ \\
& $\epsilon_{r 3}=3, \mu_{r 3}=1$ & $\chi_{\mathrm{ee}}^{z z}=1.86202 \times 10^{-2}$ \\
& $\epsilon_{r 4}=4, \mu_{r 4}=1$ & $\chi_{\mathrm{em}}^{z \phi}=j 2.78948 \times 10^{-4}$ \\
& $\epsilon_{r 5}=1, \mu_{r 5}=1$ & \\
\hline \multirow{5}{*}{ Quadradrant 3 3} & $\epsilon_{r 1}=1, \mu_{r 1}=1$ & $\chi_{\mathrm{me}}^{\phi z}=j 1.45598 \times 10^{-3}$ \\
& $\epsilon_{r 2}=4, \mu_{r 2}=1$ & $\chi_{\mathrm{mm}}^{\phi \phi}=9.25367 \times 10^{-3}$ \\
& $\epsilon_{r 3}=3, \mu_{r 3}=1$ & $\chi_{\mathrm{ee}}^{z z}=2.80732 \times 10^{-2}$ \\
& $\epsilon_{r 4}=6, \mu_{r 4}=2$ & $\chi_{\mathrm{em}}^{z \phi}=-j 9.74236 \times 10^{-4}$ \\
& $\epsilon_{r 5}=1, \mu_{r 5}=1$ & \\
\hline \multirow{5}{*}{ Quadrant 4} & $\epsilon_{r 1}=1, \mu_{r 1}=1$ & $\chi_{\mathrm{me}}^{\phi z}=j 2.83566 \times 10^{-4}$ \\
& $\epsilon_{r 2}=1, \mu_{r 2}=1$ & $\chi_{\mathrm{mm}}^{\phi \phi}=6.01626 \times 10^{-3}$ \\
& $\epsilon_{r 3}=6, \mu_{r 3}=2$ & $\chi_{\mathrm{ee}}^{z z}=1.44824 \times 10^{-2}$ \\
& $\epsilon_{r 4}=1, \mu_{r 4}=1$ & $\chi_{\mathrm{em}}^{z \phi}=-j 3.78786 \times 10^{-5}$ \\
& $\epsilon_{r 5}=1, \mu_{r 5}=1$ & \\
\hline \multicolumn{2}{|c}{}
\end{tabular}

TABLE I

Material properties and susceptibility tensor of metasurface segments shown in Fig 4. Permittivity and permeability have subscripts which represent corresponding regions.

IE-GSTC and full wave results obtained using COMSOL software can be seen. For the IE-GSTC result a small gap can be observed around the metasurface radius of $x=5 \lambda$. This is because IE results is valid only for regions interior and exterior to the metasurface. Minor discrepancies between the two results can be seen around the metasurface. This is probably due to MoM being inefficient in the near field of the scatterer as well as GSTC being an approximate interface condition. In Fig. 5 and Fig. 6, the results from the zerothickness IE-GSTC [10] are plotted for the same problem to show that the formulation in this paper is required to get more accurate results. COMSOL discretizes and solves the fields inside the thin dielectric layers, while IE-GSTC abstracts it.

In table I, it can be seen that even though the metasurface elements are passive, lossless and reciprocal the condition 


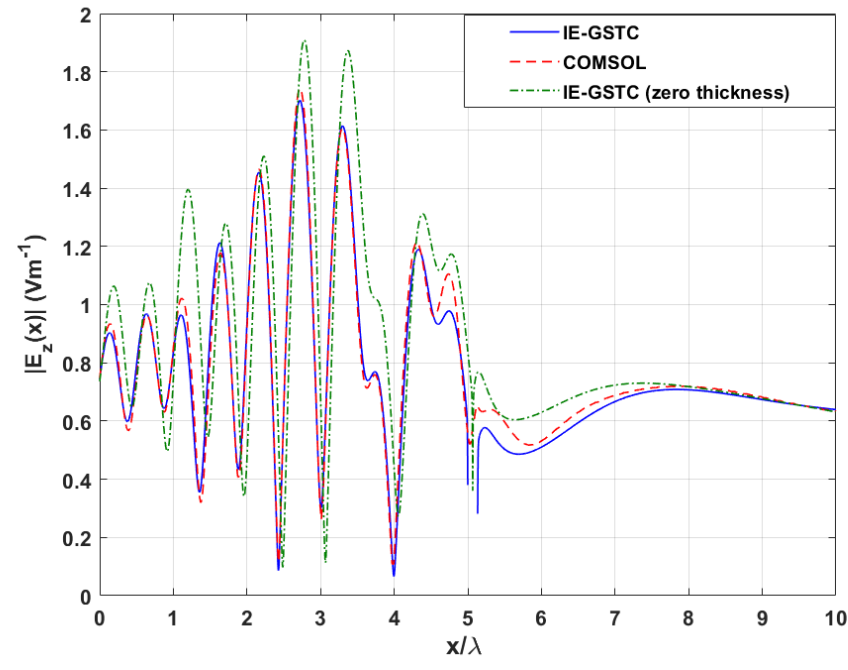

Fig. 5. IE-GSTC vs. full wave simulation for example A. $\left|E_{z}(x)\right|$ vs. $x$ from $(0,0)$ to $(10 \lambda, 0)$

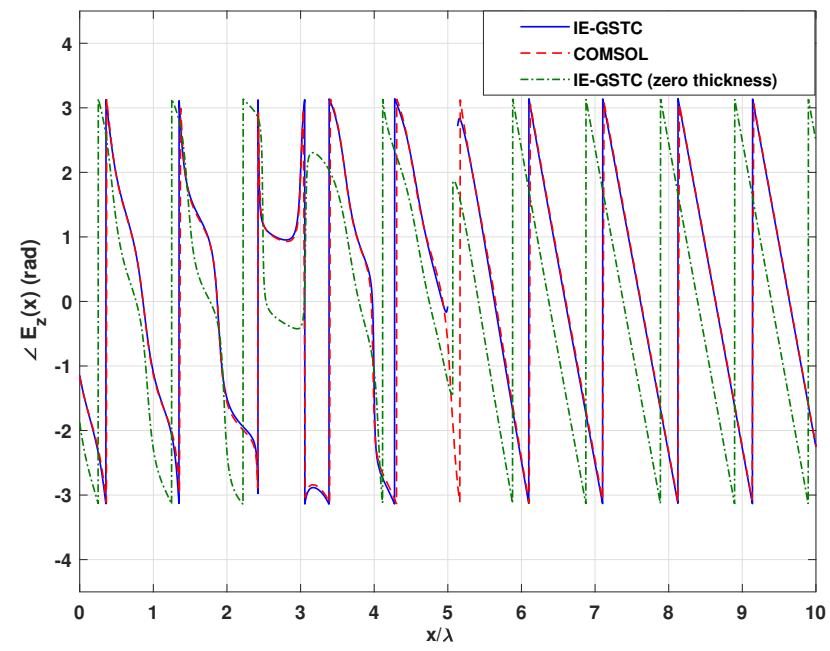

Fig. 6. IE-GSTC vs. full wave simulation for example A. $\angle E_{z}(x)$ vs. $x$ from $(0,0)$ to $(10 \lambda, 0)$

$\chi_{\mathrm{me}}^{\phi z}=-\chi_{\mathrm{em}}^{z \phi}$ as stated in [14] is not satisfied. In [14], fig. 4 shows a unit cell characterization system in which a curved, spherical metasurface particle is illuminated by both inward propagating and outward propagating spherical waves. However, [14] provides no details on the numerical implementation of such a system. The susceptibility extraction technique in this paper is essentially an analytical, cylindrical version of the numerical, spherical system shown in fig. 4 in [14]. The condition $\chi_{\mathrm{me}}^{\phi z}=-\chi_{\mathrm{em}}^{z \phi}$ is not satisfied because of the curvature, that is the inner contour of the scattering particle is not of the same length as of the outer contour. The inner contour length becomes equal to the outer contour length only when the radius of curvature becomes very large. This can be proved numerically with the susceptibility extraction method described in the last section. In table II, the susceptibility

\begin{tabular}{|c|c|}
\hline Metasurface radius & Susceptibility tensor \\
\hline$a_{1}=5 \lambda$ & $\begin{aligned} \chi_{\mathrm{me}}^{\phi z} & =j 2.55236 \times 10^{-3} \\
\chi_{\mathrm{em}} & =j 1.4211 \times 10^{-2}\end{aligned}$ \\
\hline$a_{1}=20 \lambda$ & $\begin{aligned} \chi_{\mathrm{me}}^{\phi z} & =-j 2.4572 \times 10^{-3} \\
z \phi & =j 6.7137 \times 10^{-3}\end{aligned}$ \\
\hline$a_{1}=100 \lambda$ & $\begin{array}{l}\chi_{\mathrm{me}}^{\phi z}=-j 3.8152 \times 10^{-3} \\
\chi_{\mathrm{em}}^{z \phi}=j 4.6690 \times 10^{-3}\end{array}$ \\
\hline$a_{1}=300 \lambda$ & $\begin{aligned} \chi_{\mathrm{me}}^{\phi z} & =-j 4.0421 \times 10^{-3} \\
\chi_{\mathrm{em}}^{\phi} & =j 4.3268 \times 10^{-3}\end{aligned}$ \\
\hline
\end{tabular}

tensor components of the metasurface element in quadrant I are shown as the inner radius $a_{1}$ is varied. It can be seen that for large radius of curvature, the condition is satisfied. The results of the susceptibility extraction are robust with respect to different illuminations. For instance, changing the value of outer surface current excitation i.e. $a_{s}$ gives the same results. Furthermore, a one dimensional cylindrical FDTD code [25] was developed to independently obtain the results. A one dimensional cylindrical FDTD with variation along the $\rho$ direction and no variation along $\phi, z$ directions will automatically take the curvature into account.

\section{B. Cylindrical metasurface with opening excited by a plane wave}

This example, as shown in Fig. 7, is a four segment metasurface but with an opening. The opening subtends an angle of $\pi / 2$ at the center and is equally divided between the first and fourth quadrants. The material properties of the quadrants are same as that of the previous example. GSTC cannot handle segments with material properties same as that of the background medium. In the IE-GSTC approach, an opening is handled by setting $\mathbf{A}\left(\bar{\rho}_{c}^{\prime}\right)=\operatorname{diag}(1,1,1,1)[10]$. This ensures that tangential fields on either side of the opening are equal. This is only an approximation because the opening has a width of fraction of a wavelength. IE-GSTC results and COMSOL results on a circle of radius $8 \lambda$ are plotted in Fig. 8 and Fig. 9.

\section{Eight segment metasurface excited by a line source}

In this example, an eight segment cylindrical metasurface with each segment subtending an angle of $\pi / 4$ is excited by an infinite electric, line source at origin, i.e. $\bar{J}(\bar{r})=$ $\delta(x) \delta(y) \hat{z} \mathrm{Am}^{-2}$. The dimension of the structure is same as that of the example $A$. The segment angular positions are $[0, \pi / 4],[\pi / 4, \pi / 2]$ and so on. The segments on the top half are exactly the same ones as in table I. The segments on the bottom half is listed in table. III. The magnitude and phase of the electric field on a circle of radius $8 \lambda$ are shown in Fig. 10 and Fig. 11 respectively. Zero thickness IE-GSTC [10] results are also superimposed. In the COMSOL full wave simulation, the line source is modeled using the external current density option on a circle of radius much smaller than wavelength $(\sim \lambda / 100)$. 


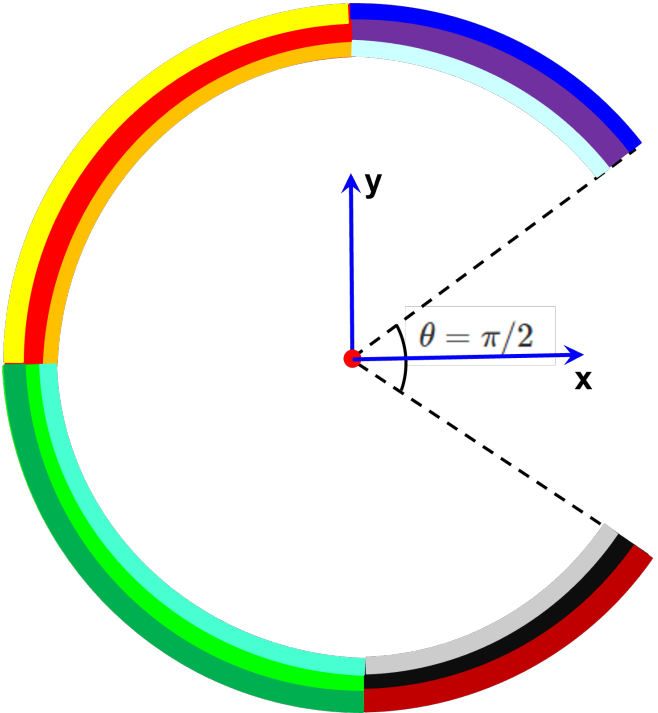

Fig. 7. Four segment cylindrical metasurface with opening

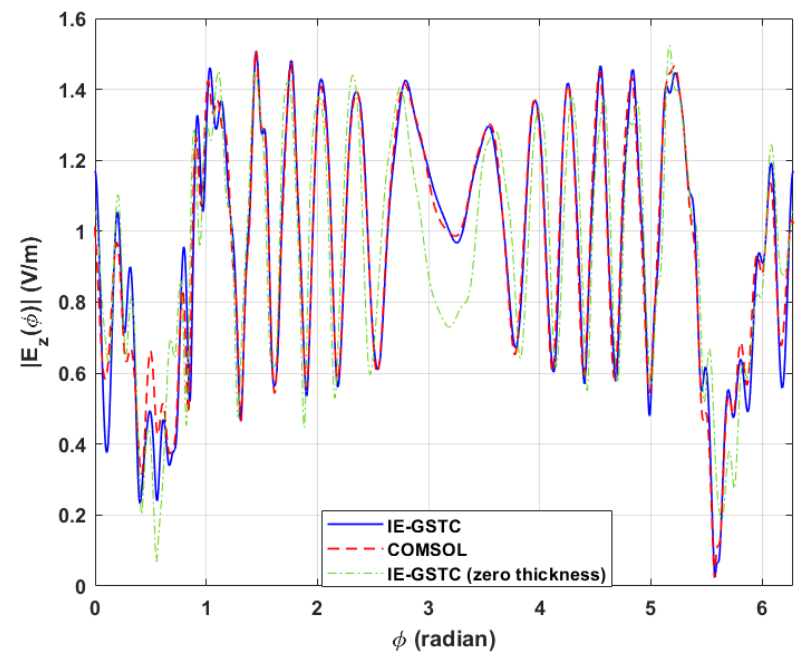

Fig. 8. IE-GSTC vs. full wave simulation for example B. $\left|E_{z}(\phi)\right|$ vs. $\phi$ on a circle of radius $8 \lambda$.

\section{IE-GSTC MODELING OF CYLINDRICAL METASURFACE - PEC COMPOSITE STRUCTURES}

In this section, the IE-GSTC formulation is extended to include PEC segments. GSTC can be used to model dielectric segments, dielectric segments with metallic/non-metallic inclusions, etc. When one of the segments is PEC, the IE-GSTC model needs to be extended. Potential applications include PEC backed metasurface lens antennas, intelligent surface enabled wireless environments [26], and scattering reduction and radiation problems [27].

The problem under consideration is shown in Fig. 12 . Similar to the previous examples, there is no variation of material properties or excitation along the $z$ axis. The inner contour is $\Gamma_{2}$ and the outer contour is $\Gamma_{1}$, separated by metasurface thickness $\delta$. The circular boundary which separates the external domain $\Omega_{1}$ and internal domain $\Omega_{2}$ is partly GSTC (mathematical abstraction for physical metasurface) and

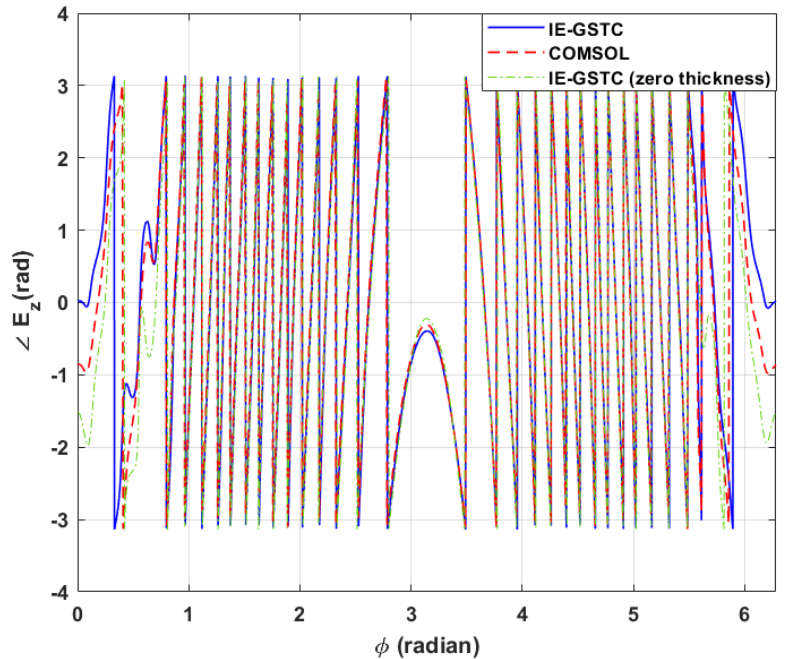

Fig. 9. IE-GSTC vs. full wave simulation for example B. $\angle E_{z}(\phi)$ vs. $\phi$ on a circle of radius $8 \lambda$.

\begin{tabular}{|c|c|c|}
\hline Location & $\begin{array}{l}\text { Material } \\
\text { properties }\end{array}$ & Susceptibility tensor \\
\hline$\pi \leq \phi<5 \pi / 4$ & $\begin{array}{l}\epsilon_{r 1}=1, \mu_{r 1}=1 \\
\epsilon_{r 2}=1, \mu_{r 2}=1 \\
\epsilon_{r 3}=4, \mu_{r 3}=1 \\
\epsilon_{r 4}=1, \mu_{r 4}=1 \\
\epsilon_{r 5}=1, \mu_{r 5}=1\end{array}$ & $\begin{array}{l}\chi_{\mathrm{me}}^{\phi z}=-j 1.50001 \times 10^{-3} \\
\chi_{\mathrm{mm}}^{\phi \phi}=4.74909 \times 10^{-3} \\
\chi_{\mathrm{ee}}^{z z}=1.62999 \times 10^{-2} \\
z \phi \\
\chi_{\mathrm{em}}=j 1.72889 \times 10^{-3}\end{array}$ \\
\hline $5 \pi / 4 \leq \phi<3 \pi / 2$ & $\begin{array}{l}\epsilon_{r 1}=1, \mu_{r 1}=1 \\
\epsilon_{r 2}=4, \mu_{r 2}=1 \\
\epsilon_{r 3}=10, \mu_{r 3}=1 \\
\epsilon_{r 4}=4, \mu_{r 4}=2 \\
\epsilon_{r 5}=1, \mu_{r 5}=1\end{array}$ & $\begin{array}{l}\chi_{\mathrm{me}}^{\phi z}=j 2.44255 \times 10^{-2} \\
\chi_{\mathrm{mm}}^{\phi \phi}=1.31683 \times 10^{-2} \\
\chi_{\mathrm{ee}}^{z z}=1.16977 \times 10^{-1} \\
\chi_{\mathrm{em}}^{z \phi}=-j 2.29491 \times 10^{-2}\end{array}$ \\
\hline $3 \pi / 2 \leq \phi<7 \pi / 4$ & $\begin{aligned} \epsilon_{r 1} & =1, \mu_{r 1}=1 \\
\epsilon_{r 2} & =1, \mu_{r 2}=1 \\
\epsilon_{r 3} & =5, \mu_{r 3}=1 \\
\epsilon_{r 4} & =1, \mu_{r 4}=1 \\
\epsilon_{r 5} & =1, \mu_{r 5}=1\end{aligned}$ & $\begin{array}{l}\chi_{\mathrm{me}}^{\phi z}=j 2.19819 \times 10^{-4} \\
\chi_{\mathrm{mm}}^{\phi \phi}=4.28646 \times 10^{-3} \\
\chi_{\mathrm{ee}}^{z z}=1.15471 \times 10^{-2} \\
\chi_{\mathrm{em}}^{z \phi}=-j 2.60041 \times 10^{-5}\end{array}$ \\
\hline $7 \pi / 4 \leq \phi<2 \pi$ & $\begin{aligned} \epsilon_{r 1} & =1, \mu_{r 1}=1 \\
\epsilon_{r 2} & =10, \mu_{r 2}=2 \\
\epsilon_{r 3} & =4, \mu_{r 3}=1 \\
\epsilon_{r 4} & =10, \mu_{r 4}=2 \\
\epsilon_{r 5} & =1, \mu_{r 5}=1\end{aligned}$ & $\begin{array}{l}\chi_{\mathrm{me}}^{\phi z}=j 3.14385 \times 10^{-2} \\
\chi_{\mathrm{mm}}^{\phi \phi}=-6.54633 \times 10^{-1} \\
\chi_{\mathrm{ee}}^{z z}=4.27841 \times 10^{-1} \\
\chi_{\mathrm{em}}^{z \phi}=-j 4.36316 \times 10^{-1}\end{array}$ \\
\hline
\end{tabular}

Material properties and susceptibility tensor of metasurface segments shown in Fig 4. Permittivity and permeability have subscripts which represent corresponding regions.

partly PEC. The GSTC part is denoted by G and the PEC part is denoted by $\mathrm{P}$. Therefore $\Gamma_{1 \mathrm{G}}$ and $\Gamma_{1 \mathrm{P}}$ represent the outer contour of GSTC and PEC sections. Similarly, $\Gamma_{2 \mathrm{G}}$ and $\Gamma_{2 \mathrm{P}}$ represent the inner contour of GSTC and PEC sections. The PEC sections do not necessarily need to be contiguous. Considering $\mathrm{TM}_{\mathrm{z}}$ polarization and referring to Fig. 12, the governing integral equations read

$$
\begin{array}{r}
\frac{E_{z 1}\left(\bar{\rho}_{1}\right)}{2}-\oint_{\Gamma_{1}}\left[E_{z 1}\left(\bar{\rho}_{1}^{\prime}\right) \frac{\partial G_{0}\left(\bar{\rho}_{1} ; \bar{\rho}_{1}^{\prime}\right)}{\partial n^{\prime}}-\right. \\
\left.j \omega \mu_{0} H_{\phi 1}\left(\bar{\rho}_{1}^{\prime}\right) G_{0}\left(\bar{\rho}_{1} ; \bar{\rho}_{1}^{\prime}\right)\right] d \Gamma_{1}^{\prime}=E_{z 1}^{\mathrm{inc}}\left(\bar{\rho}_{1}\right) ; \bar{\rho}_{1} \in \Gamma_{1}
\end{array}
$$




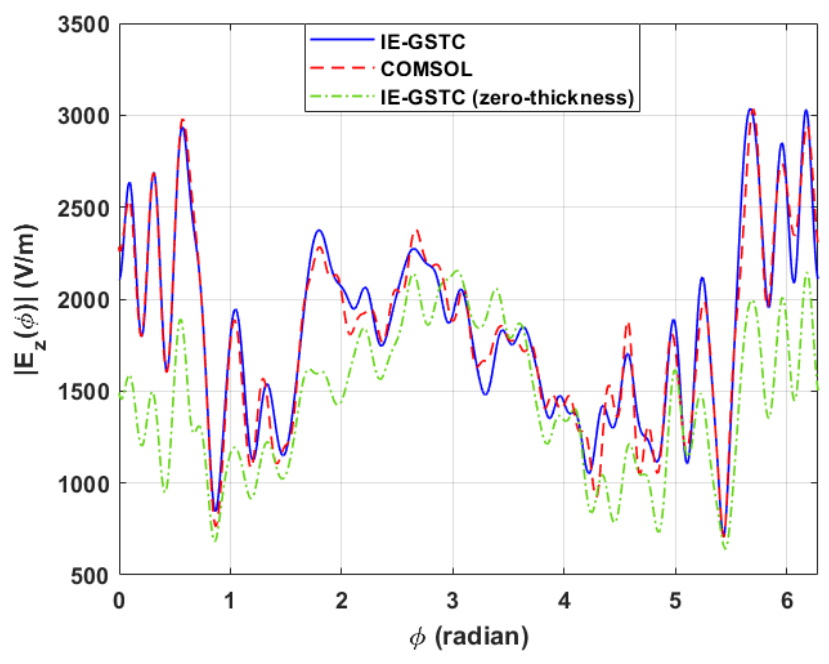

Fig. 10. IE-GSTC vs. full wave simulation for example C. $\left|E_{z}(\phi)\right|$ vs. $\phi$ on a circle of radius $8 \lambda$.

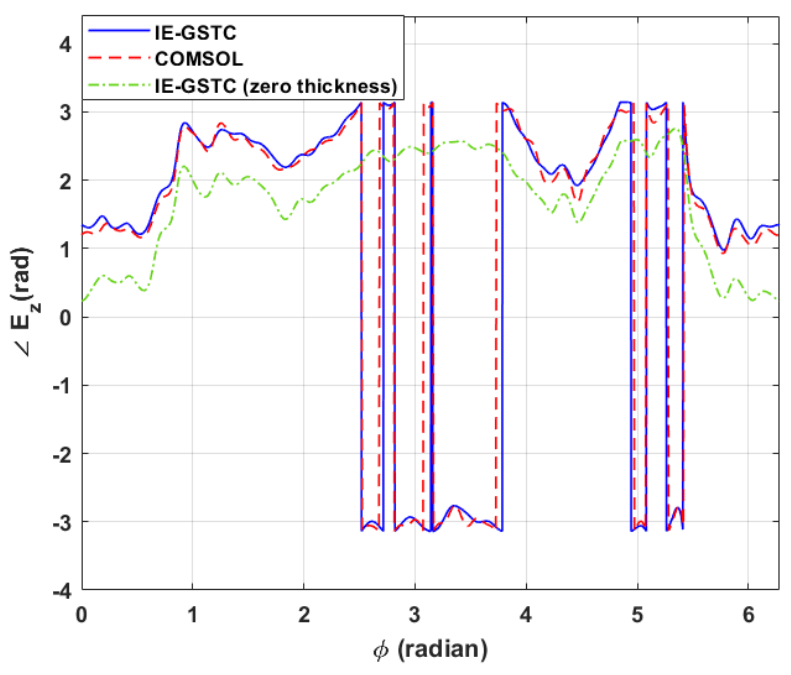

Fig. 11. IE-GSTC vs. full wave simulation for example C. $\angle E_{z}(\phi)$ vs. $\phi$ on a circle of radius $8 \lambda$.

$$
\begin{array}{r}
\frac{E_{z 2}\left(\bar{\rho}_{2}\right)}{2}+\oint_{\Gamma_{2}}\left[E_{z 2}\left(\bar{\rho}_{2}^{\prime}\right) \frac{\partial G_{0}\left(\bar{\rho}_{2} ; \bar{\rho}_{2}^{\prime}\right)}{\partial n^{\prime}}-\right. \\
\left.j \omega \mu_{0} H_{\phi 2}\left(\bar{\rho}_{2}^{\prime}\right) G_{0}\left(\bar{\rho}_{2} ; \bar{\rho}_{2}^{\prime}\right)\right] d \Gamma_{2}^{\prime}=E_{z 2}^{\mathrm{inc}}\left(\bar{\rho}_{2}\right) ; \bar{\rho}_{2} \in \Gamma_{2}
\end{array}
$$

On the GSTC part of the boundary, the external and internal tangential electric and magnetic fields are related through matrix $\mathbf{A}\left(\bar{\rho}_{c}\right)$

$$
\left[\begin{array}{l}
E_{z 2}\left(\bar{\rho}_{2}\right) \\
H_{\phi 2}\left(\bar{\rho}_{2}\right)
\end{array}\right]=\left[\begin{array}{ll}
A_{11}\left(\bar{\rho}_{c}\right) & A_{12}\left(\bar{\rho}_{c}\right) \\
A_{21}\left(\bar{\rho}_{c}\right) & A_{22}\left(\bar{\rho}_{c}\right)
\end{array}\right]\left[\begin{array}{l}
E_{z 1}\left(\bar{\rho}_{1}\right) \\
H_{\phi 1}\left(\bar{\rho}_{1}\right)
\end{array}\right]
$$

where $\bar{\rho}_{c}$ denotes the center of the metasurface, i.e. $\bar{\rho}_{c}=\bar{\rho}_{1}-$ $0.5 \delta \hat{\rho}$. For PEC segments, there is no relation between the tangential components on the inner and outer domains. By using the fact that the total tangential electric field on the

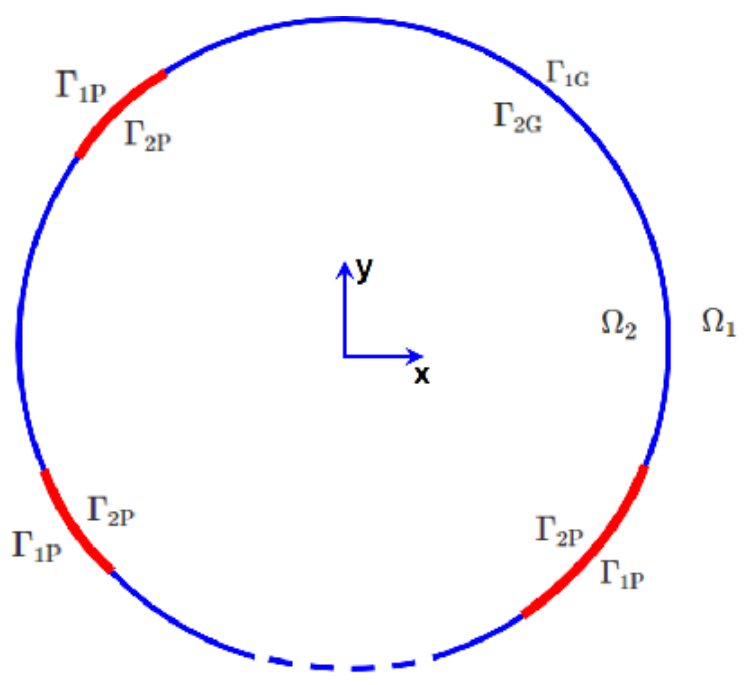

Fig. 12. Composite metasurface-PEC system

PEC surface is zero, the integral equation (20a) is converted to two IEs, one for $\bar{\rho}_{1} \in \Gamma_{1 \mathrm{G}}$ and another for $\bar{\rho}_{1} \in \Gamma_{1 \mathrm{P}}$ :

$$
\begin{array}{r}
\frac{E_{z 1}\left(\bar{\rho}_{1}\right)}{2}-\int_{\Gamma_{1 \mathrm{G}}}\left[E_{z 1}\left(\bar{\rho}_{1}^{\prime}\right) \frac{\partial G_{0}\left(\bar{\rho}_{1} ; \bar{\rho}_{1}^{\prime}\right)}{\partial n^{\prime}}-\right. \\
\left.j \omega \mu_{0} H_{\phi 1}\left(\bar{\rho}_{1}^{\prime}\right) G_{0}\left(\bar{\rho}_{1} ; \bar{\rho}_{1}^{\prime}\right)\right] d \Gamma_{1}^{\prime}-\int_{\Gamma_{1 \mathrm{P}}}\left[-j \omega \mu_{0} H_{\phi 1}\left(\bar{\rho}_{1}^{\prime}\right)\right. \\
\left.G_{0}\left(\bar{\rho}_{1} ; \bar{\rho}_{1}^{\prime}\right)\right] d \Gamma_{1}^{\prime}=E_{z 1}^{\mathrm{inc}}\left(\bar{\rho}_{1}\right) ; \bar{\rho}_{1} \in \Gamma_{1 \mathrm{G}} \\
-\int_{\Gamma_{1 \mathrm{G}}}\left[E_{z 1}\left(\bar{\rho}_{1}^{\prime}\right) \frac{\partial G_{0}\left(\bar{\rho}_{1} ; \bar{\rho}_{1}^{\prime}\right)}{\partial n^{\prime}}-\right. \\
\left.j \omega \mu_{0} H_{\phi 1}\left(\bar{\rho}_{1}^{\prime}\right) G_{0}\left(\bar{\rho}_{1} ; \bar{\rho}_{1}^{\prime}\right)\right] d \Gamma_{1}^{\prime}-\int_{\Gamma_{1 \mathrm{P}}}\left[-j \omega \mu_{0} H_{\phi 1}\left(\bar{\rho}_{1}^{\prime}\right)\right. \\
\left.G_{0}\left(\bar{\rho}_{1} ; \bar{\rho}_{1}^{\prime}\right)\right] d \Gamma_{1}^{\prime}=E_{z 1}^{\mathrm{inc}}\left(\bar{\rho}_{1}\right) ; \bar{\rho}_{1} \in \Gamma_{1 \mathrm{P}}
\end{array}
$$

By using equation (21) and the condition that the total tangential electric field on the PEC surface is zero, (20b) is transformed to two equations, one for $\bar{\rho}_{2} \in \Gamma_{2 \mathrm{G}}$ and another for $\bar{\rho}_{2} \in \Gamma_{2 \mathrm{P}}$ :

$$
\begin{array}{r}
\frac{1}{2}\left[A_{11}\left(\bar{\rho}_{c}\right) E_{z 1}\left(\bar{\rho}_{1}\right)+A_{12}\left(\bar{\rho}_{c}\right) H_{\phi 1}\left(\bar{\rho}_{1}\right)\right]+ \\
\int_{\Gamma_{2 \mathrm{G}}}\left[A_{11}\left(\bar{\rho}_{c}^{\prime}\right) E_{z 1}\left(\bar{\rho}_{1}^{\prime}\right)+A_{12}\left(\bar{\rho}_{c}^{\prime}\right) H_{\phi 1}\left(\bar{\rho}_{1}^{\prime}\right)\right] \frac{\partial G_{0}\left(\bar{\rho}_{2} ; \bar{\rho}_{2}^{\prime}\right)}{\partial n^{\prime}} d \Gamma_{2}^{\prime} \\
+\int_{\Gamma_{2 \mathrm{G}}}-j \omega \mu_{0}\left[A_{21}\left(\bar{\rho}_{c}^{\prime}\right) E_{z 1}\left(\bar{\rho}_{1}^{\prime}\right)+A_{22}\left(\bar{\rho}_{c}^{\prime}\right) H_{\phi 1}\left(\bar{\rho}_{1}^{\prime}\right)\right] \\
G_{0}\left(\bar{\rho}_{2} ; \bar{\rho}_{2}^{\prime}\right) d \Gamma_{2}^{\prime}+\int_{\Gamma_{2 \mathrm{P}}}-j \omega \mu_{0} H_{\phi 2}\left(\bar{\rho}_{2}^{\prime}\right) G_{0}\left(\bar{\rho}_{2} ; \bar{\rho}_{2}^{\prime}\right) d \Gamma_{2}^{\prime} \\
=E_{z 2}^{\mathrm{inc}}\left(\bar{\rho}_{2}\right) ; \bar{\rho}_{2} \in \Gamma_{2 \mathrm{G}}
\end{array}
$$




$$
\begin{array}{r}
\int_{\Gamma_{2 \mathrm{G}}}\left[A_{11}\left(\bar{\rho}_{c}^{\prime}\right) E_{z 1}\left(\bar{\rho}_{1}^{\prime}\right)+A_{12}\left(\bar{\rho}_{c}^{\prime}\right) H_{\phi 1}\left(\bar{\rho}_{1}^{\prime}\right)\right] \frac{\partial G_{0}\left(\bar{\rho}_{2} ; \bar{\rho}_{2}^{\prime}\right)}{\partial n^{\prime}} d \Gamma_{2}^{\prime} \\
+\int_{\Gamma_{2 \mathrm{G}}}-j \omega \mu_{0}\left[A_{21}\left(\bar{\rho}_{c}^{\prime}\right) E_{z 1}\left(\bar{\rho}_{1}^{\prime}\right)+A_{22}\left(\bar{\rho}_{c}^{\prime}\right) H_{\phi 1}\left(\bar{\rho}_{1}^{\prime}\right)\right] \\
G_{0}\left(\bar{\rho}_{2} ; \bar{\rho}_{2}^{\prime}\right) d \Gamma_{2}^{\prime}+\int_{\Gamma_{2 \mathrm{P}}}-j \omega \mu_{0} H_{\phi 2}\left(\bar{\rho}_{2}^{\prime}\right) G_{0}\left(\bar{\rho}_{2} ; \bar{\rho}_{2}^{\prime}\right) d \Gamma_{2}^{\prime} \\
=E_{z 2}^{\mathrm{inc}}\left(\bar{\rho}_{2}\right) ; \bar{\rho}_{2} \in \Gamma_{2 \mathrm{P}}
\end{array}
$$

The four equations (22a), (22b), (23b) and (23b) are used to solve for the four unknowns. The unknowns are the tangential electric and magnetic fields on $\Gamma_{1 \mathrm{G}}$, the tangential magnetic field on $\Gamma_{1 \mathrm{G}}$ and the tangential magnetic field on $\Gamma_{2 \mathrm{P}}$. These surface fields are expressed as a weighted, linear combination of pulse basis function $p_{\mathrm{n}}(\cdot)$.

$$
\begin{aligned}
& E_{z 1}\left(\bar{\rho}_{1}^{\prime}\right)=\sum_{\mathrm{n}=1}^{\mathrm{N}_{\mathrm{G}}} E_{z 1 \mathrm{G}, \mathrm{n}} p_{\mathrm{n}}\left(\bar{\rho}_{1}^{\prime}\right) ; \bar{\rho}_{1}^{\prime} \in \Gamma_{1 \mathrm{G}} \\
& H_{\phi 1}\left(\bar{\rho}_{1}^{\prime}\right)=\sum_{\mathrm{n}=1}^{\mathrm{N}_{\mathrm{G}}} H_{\phi 1 \mathrm{G}, \mathrm{n}} p_{\mathrm{n}}\left(\bar{\rho}_{1}^{\prime}\right) ; \bar{\rho}_{1}^{\prime} \in \Gamma_{1 \mathrm{G}} \\
& H_{\phi 1}\left(\bar{\rho}_{1}^{\prime}\right)=\sum_{\mathrm{n}=1}^{\mathrm{N}_{\mathrm{P}}} H_{\phi 1 \mathrm{P}, \mathrm{n}} p_{\mathrm{n}}\left(\bar{\rho}_{1}^{\prime}\right) ; \bar{\rho}_{1}^{\prime} \in \Gamma_{1 \mathrm{P}} \\
& H_{\phi 2}\left(\bar{\rho}_{2}^{\prime}\right)=\sum_{\mathrm{n}=1}^{\mathrm{N}_{\mathrm{P}}} H_{\phi 2 \mathrm{P}, \mathrm{n}} p_{\mathrm{n}}\left(\bar{\rho}_{2}^{\prime}\right) ; \bar{\rho}_{2}^{\prime} \in \Gamma_{2 \mathrm{P}}
\end{aligned}
$$

where $N_{G}, N_{P}$ are the number of basis functions for the GSTC and PEC parts, respectively, on either the inner or outer surface. Therefore the total number of unknowns are $2\left(\mathrm{~N}_{\mathrm{G}}+\mathrm{N}_{\mathrm{P}}\right)$. By using the above expansions and testing functions $\delta\left(\bar{\rho}_{1}-\bar{\rho}_{1 \mathrm{G}, \mathrm{m}}\right), \mathrm{m}=1,2, \cdots, \mathrm{N}_{\mathrm{G}}$ for $(22 \mathrm{a}), \delta\left(\bar{\rho}_{1}-\right.$ $\left.\bar{\rho}_{1 \mathrm{P}, \mathrm{m}}\right), \mathrm{m}=1,2, \cdots, \mathrm{N}_{\mathrm{P}}$ for $(22 \mathrm{~b}), \delta\left(\bar{\rho}_{2}-\bar{\rho}_{2 \mathrm{G}, \mathrm{m}}\right), \mathrm{m}=$ $1,2, \cdots, \mathrm{N}_{\mathrm{G}}$ for (23a) and $\delta\left(\bar{\rho}_{2}-\bar{\rho}_{2 \mathrm{P}, \mathrm{m}}\right), \mathrm{m}=1,2, \cdots, \mathrm{N}_{\mathrm{P}}$ for (23b) an MoM system of equations can be derived. The MoM system of linear equations reads

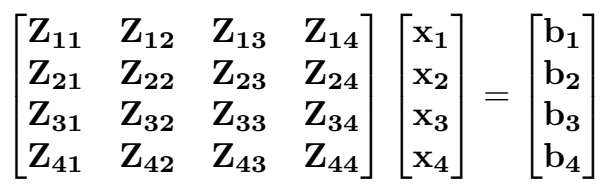

where the unknown vectors are

$$
\begin{aligned}
& \mathbf{x}_{\mathbf{1}}=\left[E_{\mathrm{z} 1 \mathrm{G}, 1}, \cdots, E_{\mathrm{z} 1 \mathrm{G}, \mathrm{NG}}\right]^{\mathrm{T}} \\
& \mathbf{x}_{\mathbf{2}}=\left[H_{\phi 1 \mathrm{G}, 1}, \cdots, H_{\phi 1 \mathrm{G}, \mathrm{NG}}\right]^{\mathrm{T}} \\
& \mathbf{x}_{\mathbf{3}}=\left[H_{\phi 1 \mathrm{P}, 1}, \cdots, H_{\phi 1 \mathrm{P}, \mathrm{NP}}\right]^{\mathrm{T}} \\
& \mathbf{x}_{\mathbf{4}}=\left[H_{\phi 2 \mathrm{P}, 1}, \cdots, H_{\phi 2 \mathrm{P}, \mathrm{NP}}\right]^{\mathrm{T}}
\end{aligned}
$$

The $\mathbf{Z}$ matrix coefficients and the excitation vector are as follows

$$
\begin{array}{ll}
Z_{11, \mathrm{mn}}=p_{\mathrm{mn}}^{1 \mathrm{G} 1 \mathrm{G}} ; & \left(\mathrm{N}_{\mathrm{G}} \times \mathrm{N}_{\mathrm{G}}\right) \\
Z_{12, \mathrm{mn}}=r_{\mathrm{mn}}^{1 \mathrm{G} 1 \mathrm{G}} ; & \left(\mathrm{N}_{\mathrm{G}} \times \mathrm{N}_{\mathrm{G}}\right) \\
Z_{13, \mathrm{mn}}=r_{\mathrm{mn}}^{1 \mathrm{G} 1 \mathrm{P}} ; & \left(\mathrm{N}_{\mathrm{G}} \times \mathrm{N}_{\mathrm{P}}\right) \\
Z_{14, \mathrm{mn}}=0 \quad ;\left(\mathrm{N}_{\mathrm{G}} \times \mathrm{N}_{\mathrm{P}}\right)
\end{array}
$$

$$
\begin{aligned}
Z_{21, \mathrm{mn}}=-t_{\mathrm{mn}}^{1 \mathrm{P} 1 \mathrm{G}} ;\left(\mathrm{N}_{\mathrm{P}} \times \mathrm{N}_{\mathrm{G}}\right) \\
Z_{22, \mathrm{mn}}=r_{\mathrm{mn}}^{1 \mathrm{P} 1 \mathrm{G}} ;\left(\mathrm{N}_{\mathrm{P}} \times \mathrm{N}_{\mathrm{G}}\right) \\
Z_{23, \mathrm{mn}}=r_{\mathrm{mn}}^{1 \mathrm{P} 1 \mathrm{P}} ;\left(\mathrm{N}_{\mathrm{P}} \times \mathrm{N}_{\mathrm{P}}\right) \\
Z_{24, \mathrm{mn}}=0 \quad ;\left(\mathrm{N}_{\mathrm{P}} \times \mathrm{N}_{\mathrm{P}}\right) \\
Z_{31, \mathrm{mn}=} A_{11, \mathrm{n}} q_{\mathrm{mn}}^{2 \mathrm{G} 2 \mathrm{G}}-A_{21, \mathrm{n}} r_{\mathrm{mn}}^{2 \mathrm{G} 2 \mathrm{G}} ;\left(\mathrm{N}_{\mathrm{G}} \times \mathrm{N}_{\mathrm{G}}\right) \\
Z_{32, \mathrm{mn}}=A_{12, \mathrm{n}} q_{\mathrm{mn}}^{2 \mathrm{G} 2 \mathrm{G}}-A_{22, \mathrm{n}} r_{\mathrm{mn}}^{2 \mathrm{G} 2 \mathrm{G}} ;\left(\mathrm{N}_{\mathrm{G}} \times \mathrm{N}_{\mathrm{G}}\right) \\
Z_{33, \mathrm{mn}=}=0 \quad ;\left(\mathrm{N}_{\mathrm{G}} \times \mathrm{N}_{\mathrm{P}}\right) \\
Z_{34, \mathrm{mn}=}=r_{\mathrm{mn}}^{2 \mathrm{G} 2 \mathrm{P}} ;\left(\mathrm{N}_{\mathrm{G}} \times \mathrm{N}_{\mathrm{P}}\right) \\
Z_{41, \mathrm{mn}}=A_{11, \mathrm{n}} t_{\mathrm{mn}}^{2 \mathrm{P} 2 \mathrm{G}}-A_{21, \mathrm{n}} r_{\mathrm{mn}}^{2 \mathrm{P} 2 \mathrm{G}} ;\left(\mathrm{N}_{\mathrm{P}} \times \mathrm{N}_{\mathrm{G}}\right) \\
Z_{42, \mathrm{mn}}=A_{12, \mathrm{n}} t_{\mathrm{mn}}^{2 \mathrm{P} 2 \mathrm{G}}-A_{22, \mathrm{n}} r_{\mathrm{mn}}^{2 \mathrm{P} 2 \mathrm{G}} ;\left(\mathrm{N}_{\mathrm{P}} \times \mathrm{N}_{\mathrm{G}}\right) \\
Z_{43, \mathrm{mn}}=0 \quad ;\left(\mathrm{N}_{\mathrm{P}} \times \mathrm{N}_{\mathrm{P}}\right) \\
Z_{44, \mathrm{mn}}=-r_{\mathrm{mn}}^{2 \mathrm{P} 2 \mathrm{P}} ;\left(\mathrm{N}_{\mathrm{P}} \times \mathrm{N}_{\mathrm{P}}\right)
\end{aligned}
$$

where $A_{\mathrm{ij}, \mathrm{n}}$ is the value of matrix element $A_{\mathrm{ij}}$ at the $n^{\text {th }}$ discretization segment. The entries in the matrix elements are calculated as follows:

$$
\begin{aligned}
& p_{\mathrm{mn}}^{1 \mathrm{G} 1 \mathrm{G}}=\frac{1}{2} \delta_{\mathrm{mn}}-\int_{s_{1 \mathrm{G}, \mathrm{n}}} \frac{\partial G_{0}\left(\bar{\rho}_{1 \mathrm{G}, \mathrm{m}} ; \bar{\rho}_{1}^{\prime}\right)}{\partial n^{\prime}} d \Gamma_{1}^{\prime} \\
& r_{\mathrm{mn}}^{1 \mathrm{G} 1 \mathrm{G}}=j \omega \mu_{0} \int_{s_{1 \mathrm{G}, \mathrm{n}}} G_{0}\left(\bar{\rho}_{1 \mathrm{G}, \mathrm{m}} ; \bar{\rho}_{1}^{\prime}\right) d \Gamma_{1}^{\prime} \\
& r_{\mathrm{mn}}^{1 \mathrm{G} 1 \mathrm{P}}=j \omega \mu_{0} \int_{s_{1 \mathrm{P}, \mathrm{n}}} G_{0}\left(\bar{\rho}_{1 \mathrm{G}, \mathrm{m}} ; \bar{\rho}_{1}^{\prime}\right) d \Gamma_{1}^{\prime} \\
& t_{\mathrm{mn}}^{1 \mathrm{P} 1 \mathrm{G}}=\int_{s_{1 \mathrm{G}, \mathrm{n}}} \frac{\partial G_{0}\left(\bar{\rho}_{1 \mathrm{P}, \mathrm{m}} ; \bar{\rho}_{1}^{\prime}\right)}{\partial n^{\prime}} d \Gamma_{1}^{\prime} \\
& r_{\mathrm{mn}}^{1 \mathrm{P} 1 \mathrm{G}}=j \omega \mu_{0} \int_{s_{1 \mathrm{G}, \mathrm{n}}} G_{0}\left(\bar{\rho}_{1 \mathrm{P}, \mathrm{m}} ; \bar{\rho}_{1}^{\prime}\right) d \Gamma_{1}^{\prime} \\
& r_{\mathrm{mn}}^{1 \mathrm{P} 1 \mathrm{P}}=j \omega \mu_{0} \int_{s_{1 \mathrm{P}, \mathrm{n}}} G_{0}\left(\bar{\rho}_{1 \mathrm{P}, \mathrm{m}} ; \bar{\rho}_{1}^{\prime}\right) d \Gamma_{1}^{\prime} \\
& q_{\mathrm{mn}}^{2 \mathrm{G} 2 \mathrm{G}}=\frac{1}{2} \delta_{\mathrm{mn}}+\int_{s_{2 \mathrm{G}, \mathrm{n}}} \frac{\partial G_{0}\left(\bar{\rho}_{2 \mathrm{G}, \mathrm{m}} ; \bar{\rho}_{2}^{\prime}\right)}{\partial n^{\prime}} d \Gamma_{2}^{\prime} \\
& r_{\mathrm{mn}}^{2 \mathrm{G} 2 \mathrm{G}}=j \omega \mu_{0} \int_{s_{2 \mathrm{G}, \mathrm{n}}} G_{0}\left(\bar{\rho}_{2 \mathrm{G}, \mathrm{m}} ; \bar{\rho}_{2}^{\prime}\right) d \Gamma_{2}^{\prime} \\
& r_{\mathrm{mn}}^{2 \mathrm{G} 2 \mathrm{P}}=j \omega \mu_{0} \int_{s_{2 \mathrm{P}, \mathrm{n}}} G_{0}\left(\bar{\rho}_{2 \mathrm{G}, \mathrm{m}} ; \bar{\rho}_{2}^{\prime}\right) d \Gamma_{2}^{\prime} \\
& t_{\mathrm{mn}}^{2 \mathrm{P} 2 \mathrm{G}}=\int_{s_{2 \mathrm{G}, \mathrm{n}}} \frac{\partial G_{0}\left(\bar{\rho}_{2 \mathrm{P}, \mathrm{m}} ; \bar{\rho}_{2}^{\prime}\right)}{\partial n^{\prime}} d \Gamma_{2}^{\prime} \\
& r_{\mathrm{mn}}^{2 \mathrm{P} 2 \mathrm{G}}=j \omega \mu_{0} \int_{s_{2 \mathrm{G}, \mathrm{n}}} G_{0}\left(\bar{\rho}_{2 \mathrm{P}, \mathrm{m}} ; \bar{\rho}_{2}^{\prime}\right) d \Gamma_{2}^{\prime} \\
& r_{\mathrm{mn}}^{2 \mathrm{P} 2 \mathrm{P}}=j \omega \mu_{0} \int_{s_{2 \mathrm{P}, \mathrm{n}}} G_{0}\left(\bar{\rho}_{2 \mathrm{P}, \mathrm{m}} ; \bar{\rho}_{2}^{\prime}\right) d \Gamma_{2}^{\prime}
\end{aligned}
$$

where $s_{n 1}, s_{n 2}$ denote the $n^{\text {th }}$ discretization segments on contours $\Gamma_{1}, \Gamma_{2}$ respectively. $\bar{\rho}_{m 1}, \bar{\rho}_{m 2}$ are the middle points of the $m^{\text {th }}$ segments on contours $\Gamma_{1}$ and $\Gamma_{2}$, respectively. $\delta_{m n}$ is the Kronecker delta function. The first part of superscript in the above coefficients denotes whether the testing is performed on the inner or outer contour. It also shows whether the testing is done on the GSTC part or PEC part. The second part of the superscript denotes whether the integration is performed in the 
outer or inner contour's GSTC part or PEC part. For example in $t_{\mathrm{mn}}^{\mathrm{P} 1 \mathrm{G}}$, the testing is done on the exterior contour's PEC part (i.e. 1P) and the integration is performed on the exterior contour's GSTC part (i.e. 1G).

The excitation vector components are given by

$$
\begin{aligned}
& b_{1, m}=E_{z 1}^{\mathrm{inc}}\left(\bar{\rho}_{m 1 G}\right), b_{2, m}=E_{z 1}^{\mathrm{inc}}\left(\bar{\rho}_{m 1 P}\right) \\
& b_{3, m}=E_{z 2}^{\mathrm{inc}}\left(\bar{\rho}_{m 2 G}\right), b_{4, m}=E_{z 2}^{\mathrm{inc}}\left(\bar{\rho}_{m 2 P}\right)
\end{aligned}
$$

These four excitation vectors provide the possibility for $\mathrm{TM}_{\mathrm{z}}$ excitation internal and external to the metasurface-PEC system. To validate the formulation, consider the metasurfacePEC system example shown in Fig. 13. The four metasurface segments are identical to what is shown in table I. The first quadrant in table I corresponds to a metasurface segment in the range $0 \leq \phi \leq \pi / 4$ in Fig. 13 and so on. The dimensions are same as used in previous examples. The excitation is a plane wave $\bar{E}^{\text {inc }}(x)=e^{-j k_{0} x} \hat{z}$. In Fig. 14 and Fig. 15, IEGSTC-PEC results are compared with COMSOL full wave simulations on a circle of radius $10 \lambda$.

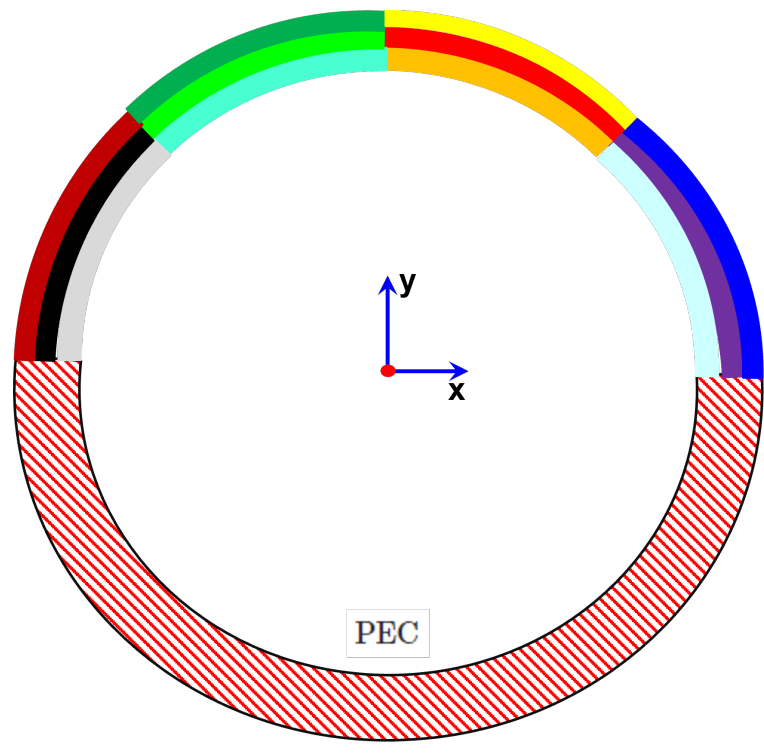

Fig. 13. Four segment metasurface with PEC backing

\section{CONCLUSION}

This work validates IE-GSTC for circular cylindrical metasurfaces of nonzero thickness. The essence of this work is that physical circular metasurface scattering particles can be abstracted by a few numbers, i.e., susceptibility tensor components. To the author's knowledge, this work is one of the few works in which the GSTC and a bianisotropic susceptibility tensor model is validated for a physical, conformal metasurface. Although the examples and susceptibility extraction are presented for the TM polarization, it is easily extendable to the TE polarization. The combination can be used to analyze and design circular metasurfaces for the case of circularly polarized incident waves. This work is important in the sense that it pushes the previous works on conformal GSTC [10][12], [14] to physical implementation and optimization. The IE-GSTC formulation is extended to handle PEC segments which could be useful for

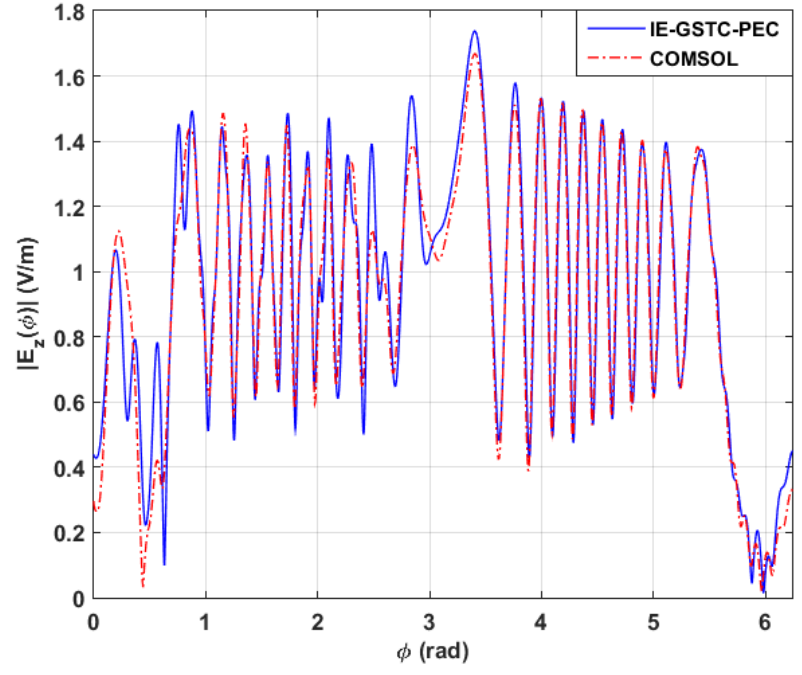

Fig. 14. IE-GSTC-PEC vs. full wave simulation for four segment metasurface with PEC backing. $\left|E_{z}(\phi)\right|$ vs. $\phi$ on a circle of radius $10 \lambda$.

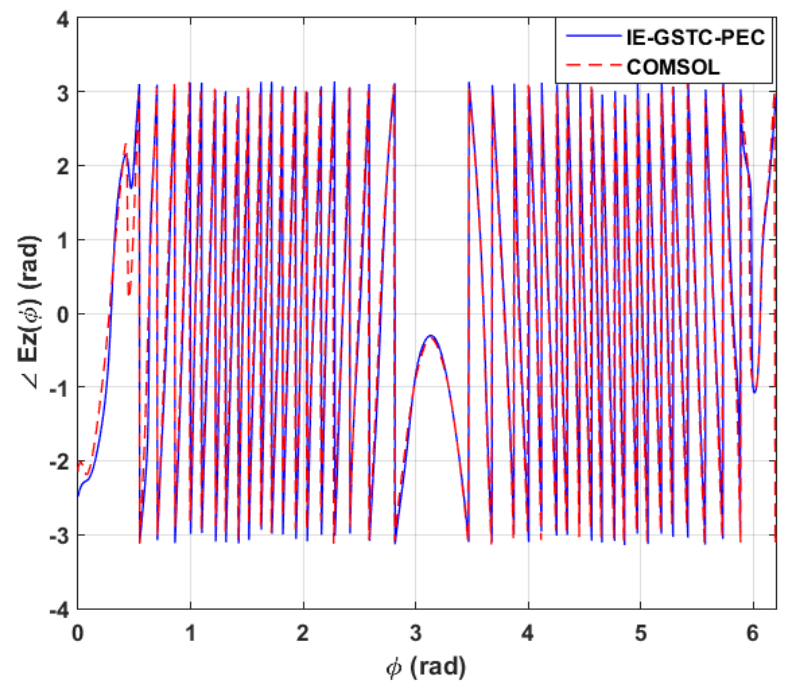

Fig. 15. IE-GSTC-PEC vs. full wave simulation for four segment metasurface with PEC backing. $\angle E_{z}(\phi)$ vs. $\phi$ on a circle of radius $10 \lambda$.

\section{APPENDIX A}

MULTILAYERED MAGNETO-DIELECTRIC SHELL INSIDE A CYLINDRICAL SURFACE ELECTRIC CURRENT DENSITY

This section provides the analytical solution to the scattering problem shown in fig. 3. The excitation is a cylindrical surface electric current density of radius $a_{s}$, represented as $\bar{J}_{s}=\delta\left(\rho-a_{s}\right) \hat{z}$. Even though fig. 3 shows a three layered shell, this section generalizes it to $m-1$ magneto-dielectric layers. The $m+2$ regions, i.e. $m-1$ magneto-dielectric regions, region internal to the shell, and space outside the shell separated into two regions by the surface current density are identified as follows:

$$
\begin{aligned}
& \text { Region } i: a_{i-1} \leq \rho \leq a_{i} ; i=1,2, \cdots, m+2 \\
& a_{0}=0 ; a_{m+1}=a_{s} ; a_{m+2}=\infty
\end{aligned}
$$


where $a_{m+1}=a_{s}$ separates the external space (outside the magneto-dielectric shell) into two regions. There is a field discontinuity between these regions due to $\bar{J}_{s}=\delta\left(\rho-a_{s}\right) \hat{z}$. Region $i$ has the constitutive parameters $\left(\epsilon_{i}, \mu_{i}\right)$ and wave number $k_{i}=\omega \sqrt{\epsilon_{i} \mu_{i}}$. Assuming no field or material variation along $\phi$ and $z$ direction, the vector magnetic potential, electric field and magnetic field in each region for the case of $\mathrm{TM}_{\mathrm{z}}$ polarization can be expressed in terms of Bessel functions.

$$
\begin{array}{r}
A_{z, i}(\rho)=C_{i} J_{0}\left(k_{i} \rho\right)+D_{i} Y_{0}\left(k_{i} \rho\right) \\
E_{z, i}(\rho)=-j \omega\left[C_{i} J_{0}\left(k_{i} \rho\right)+D_{i} Y_{0}\left(k_{i} \rho\right)\right] \\
H_{\phi, i}(\rho)=\frac{k_{i}}{\mu_{i}}\left[C_{i} J_{1}\left(k_{i} \rho\right)+D_{i} Y_{1}\left(k_{i} \rho\right)\right]
\end{array}
$$

where $J_{0}(\cdot), Y_{0}(\cdot)$ are Bessel functions of the first and second kind, respectively. Hence there are $2(m+2)$ unknowns to be determined. The singularity of $Y_{0}(\cdot)$ at $\rho=0$ and the presence of only an outgoing wave in the outermost region $m+2$, i.e. $A_{z, m+2}(\rho) \propto H_{0}^{(2)}\left(k_{m+2} \rho\right)$ entails the following conditions:

$$
\begin{aligned}
D_{1} & =0 \\
D_{m+2}+j C_{m+2} & =0
\end{aligned}
$$

The continuity of the tangential electric field across the region interfaces results in:

$$
\begin{array}{r}
C_{i} J_{0}\left(k_{i} a_{i}\right)+D_{i} Y_{0}\left(k_{i} a_{i}\right)-C_{i+1} J_{0}\left(k_{i+1} a_{i}\right) \\
-D_{i+1} Y_{0}\left(k_{i+1} a_{i+1}\right)=0 ; i=1,2, \cdots, m+1
\end{array}
$$

The continuity of the tangential magnetic field across the interfaces and the discontinuity caused by the surface electric current density excitation results in:

$$
\begin{aligned}
& C_{i} \frac{k_{i}}{\mu_{i}} J_{1}\left(k_{i} a_{i}\right)+D_{i} \frac{k_{i}}{\mu_{i}} Y_{1}\left(k_{i} a_{i}\right)-C_{i+1} \frac{k_{i+1}}{\mu_{i+1}} J_{1}\left(k_{i+1} a_{i}\right) \\
& -D_{i+1} \frac{k_{i+1}}{\mu_{i+1}} Y_{1}\left(k_{i+1} a_{i}\right)=\delta_{i, m+1} ; i=1,2, \cdots, m+1
\end{aligned}
$$

The set of equations (35), (36) and (37) constitute $2(m+2)$ equations that can be solved for fields everywhere.

\section{REFERENCES}

[1] C. L. Holloway, E. F. Kuester, J. A. Gordon, J. O. Hara, J. Booth, and D. R. Smith, "An overview of the theory and applications of metasurfaces: The two-dimensional equivalents of metamaterials," IEEE Antennas Propag. Mag., vol. 54, no. 2, pp. 10-35, Apr 2012.

[2] S. B. Glybovski, S. A. Tretyakov, P. A. Belov, Y. S. Kivshar, and C. R. Simovski, "Metasurfaces: From microwaves to visible," Physics Reports, vol. 634, pp. $1-72$, 2016, metasurfaces: From microwaves to visible.

[3] F. Yang and Y. Rahmat-Samii, Surface Electromagnetics: With Applications in Antenna, Microwave, and Optical Engineering. Cambridge University Press, 2019.

[4] K. Achouri and C. Caloz, Electromagnetic Metasurfaces: Theory and Applications. John Wiley and Sons, 2021.

[5] M. Idemen, Discontinuities in the Electromagnetic Field, 1st ed. John Wiley \& Sons, 2011.

[6] E. F. Kuester, M. A. Mohamed, M. Piket-May, and C. L. Holloway, "Averaged transition conditions for electromagnetic fields at a metafilm," IEEE Transactions on Antennas and Propagation, vol. 51, no. 10, pp. 2641-2651, 2003.

[7] K. Achouri, M. A. Salem, and C. Caloz, "General metasurface synthesis based on susceptibility tensors," IEEE Trans. Antennas Propagat., vol. 63, no. 7, pp. 2977-2991, 2015.

[8] Y. Vahabzadeh, K. Achouri, and C. Caloz, "Simulation of metasurfaces in finite difference techniques," IEEE Transactions on Antennas and Propagation, vol. 64, no. 11, pp. 4753-4759, Nov 2016.
[9] S. Sandeep, J. M. Jin, and C. Caloz, "Finite-element modeling of metasurfaces with generalized sheet transition conditions," IEEE Transactions on Antennas and Propagation, vol. 65, no. 5, pp. 2413-2420, May 2017.

[10] S. Sandeep and S. Y. Huang, "Simulation of circular cylindrical metasurfaces using GSTC-MOM," IEEE Journal on Multiscale and Multiphysics Computational Techniques, vol. 3, pp. 185-192, 2018.

[11] M. Dehmollaian, N. Chamanara, and C. Caloz, "Wave scattering by a cylindrical metasurface cavity of arbitrary cross section: Theory and applications," IEEE Transactions on Antennas and Propagation, vol. 67, no. 6, pp. 4059-4072, 2019.

[12] M. Dehmollaian and C. Caloz, "Perfect penetrable cloaking using gainless and loss-less bianisotropic metasurfaces," in 2019 IEEE International Symposium on Antennas and Propagation and USNC-URSI Radio Science Meeting, 2019, pp. 1323-1324.

[13] M. Safari, H. Kazemi, A. Abdolali, M. Albooyeh, and F. Capolino, "Illusion mechanisms with cylindrical metasurfaces: A general synthesis approach," Phys. Rev. B, vol. 100, p. 165418, Oct 2019. [Online]. Available: https://link.aps.org/doi/10.1103/PhysRevB.100.165418

[14] X. Jia, Y. Vahabzadeh, C. Caloz, and F. Yang, "Synthesis of spherical metasurfaces based on susceptibility tensor gstcs," IEEE Transactions on Antennas and Propagation, vol. 67, no. 4, pp. 2542-2554, 2019.

[15] S. Sandeep and S. Y. Huang, "Fast analysis of spherical metasurfaces using vector wave function expansion," IEEE Antennas and Wireless Propagation Letters, vol. 18, no. 6, pp. 1086-1090, 2019.

[16] H. Li, C. Ma, F. Shen, K. Xu, D. Ye, J. Huangfu, C. Li, L. Ran, and T. A. Denidni, "Wide-angle beam steering based on an active conformal metasurface lens," IEEE Access, vol. 7, pp. 185 264-185 272, 2019.

[17] G. Xu, G. V. Eleftheriades, and S. V. Hum, "Approach to the analysis and synthesis of cylindrical metasurfaces with noncircular cross sections based on conformal transformations," Phys. Rev. B, vol. 102, p. 245305, Dec 2020. [Online]. Available: https://link.aps.org/doi/10.1103/PhysRevB.102.245305

[18] — "Discrete-fourier-transform-based framework for analysis and synthesis of cylindrical Omega-bianisotropic metasurfaces," Phys. Rev. Applied, vol. 14, p. 064055, Dec 2020. [Online]. Available: https://link.aps.org/doi/10.1103/PhysRevApplied.14.064055

[19] T. Brown, C. Narendra, Y. Vahabzadeh, C. Caloz, and P. Mojabi, "On the use of electromagnetic inversion for metasurface design," IEEE Transactions on Antennas and Propagation, vol. 68, no. 3, pp. 18121824, 2020.

[20] S. Stewart, Y. L. C. de Jong, T. J. Smy, and S. Gupta, "Rayoptical evaluation of scattering from electrically large metasurfaces characterized by locally periodic surface susceptibilities," 2021. [Online]. Available: https://arxiv.org/abs/2102.07041

[21] K. Wang, Q. Zhang, and Q. Zhang, "Electromagnetic simulation of 2.5dimensional cylindrical metasurfaces with arbitrary shapes using gstcmfcm," IEEE Access, vol. 8, pp. 142 101-142 110, 2020.

[22] S. A. Stewart, S. Moslemi-Tabrizi, T. J. Smy, and S. Gupta, "Scattering field solutions of metasurfaces based on the boundary element method for interconnected regions in 2-d," IEEE Transactions on Antennas and Propagation, vol. 67, no. 12, pp. 7487-7495, 2019.

[23] T. J. Smy and S. Gupta, "Surface susceptibility synthesis of metasurface skins/holograms for electromagnetic camouflage/illusions," IEEE Access, vol. 8, pp. 226866-226886, 2020.

[24] J.-M. Jin, Theory and computation of electromagnetic fields, 2nd ed. Wiley-IEEE Press, 2015.

[25] U. S. Inan and R. A. Marshall, Numerical electromagnetics - The FDTD method. Cambridge University Press, 2011.

[26] J. Dugan, T. J. Smy, and S. Gupta, "Accelerated IE-GSTC solver for large-scale metasurface field scattering problems using fast multipole method (FMM)," Techrxiv, 2021.

[27] B. Thtreswar, A. Díaz-Rubio, V. Asadchy, H. Ouslimani, and S. Tretyakov, "Step-wise homogeneous passive coatings for reduction of electromagnetic scattering from cylindrical metallic bodies," Journal of Optics, vol. 22, 072020. 


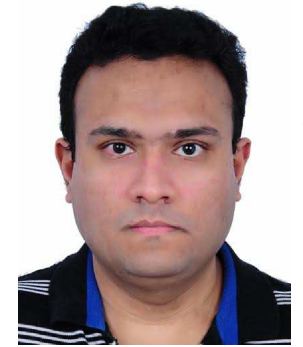

Srikumar Sandeep (M'12) received his M.S. and $\mathrm{Ph} . \mathrm{D}$. degree in electrical engineering from University of Colorado, Boulder in 2011 and 2012 respectively. He had done postdoctoral work at University of Colorado, Boulder in 2013 on antenna design, Polytechnique, Montreal in 2016 on metasurfaces and Singapore University of Technology and Design (SUTD) in 2018. He has several years of industrial experience in software development, electronics engineering and signal integrity at several companies including Trimble Navigation Ltd (San Jose) and Qualcomm Inc (San Diego). He is currently a scientist at NTNU, Trondheim, Norway. His technical interests include applied and computational electromagnetics (FDTD, MoM and 2D FEM), antenna design, hardware software development, signal integrity, wireless power transfer, software / hardware development, applied physics and applied machine learning.

He served as a reviewer for several journals such as Applied Optics, IEEE Antennas and Wireless Propagation Letters, Applied Computational Electromagnetics Society (ACES) Journal, and IEEE Transactions on Geoscience and Remote Sensing. He has more than 20 publications and a patent.

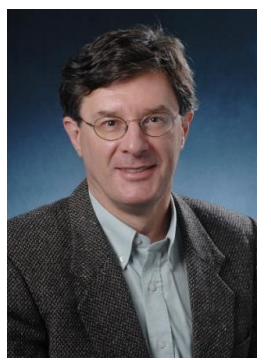

Albin J. Gasiewski (StM`81 M`88, SM`95, F`02) is a Professor of Electrical and Computer Engineering at the University of Colorado at Boulder and Director of the CU Center for Environmental Technology (CET). He received the Ph.D. degree in electrical engineering and computer science from the Massachusetts Institute of Technology in 1989. Previously, he received the M.S. and B.S. degrees in electrical engineering and the B.S. degree in mathematics from Case Western Reserve University in 1983. From 1989 to 1997 he was a faculty member at the Georgia Institute of Technology, in Atlanta GA. From 1997 through 2005 he worked for the U.S. National Oceanic and Atmospheric Administration's (NOAA) Environmental Technology Laboratory in Boulder, Colorado, USA, where he became Chief of ETL's Microwave Systems Development Division. He has developed and taught graduate courses on electromagnetics, antennas, remote sensing, instrumentation, statistical signal processing and wave propagation theory. Prof. Gasiewski is a Co-founder and Chief Scientist of Orbital Micro Systems, Inc.

Prof. Gasiewski is a Fellow of the IEEE, Past President (2004-2005) of the IEEE Geoscience and Remote Sensing Society, and founding member of the IEEE Committee on Earth Observation (ICEO). He is a member of the American Meteorological Society, the American Geophysical Union, the International Union of Radio Scientists (URSI), Tau Beta Pi, and Sigma Xi From 2009-2011 he served as Chair of USNC/URSI Commission F. He served on the U.S. National Research Council's Committee on Radio Frequencies (CORF) from 1989-1995. He was the General Co-chair of IGARSS 2006, in Denver, Colorado, and a recipient of the 2006 Outstanding Service Award and the 2017 Education Award from the GRSS.

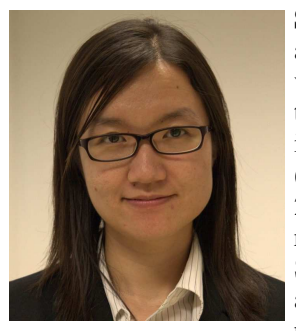

Shao Ying Huang received the B.Eng., M. Eng., and Ph.D. degree from Nanyang Technological University, Singapore in 2003, 2006, and 2011, respectively. She joined the University of Hong Kong in 2010 working on computational electromagnetics (EM), and Massachusetts Institute of Technology in 2012 working on magnetic resonance imaging (MRI) related EM problems, both as a postdoctoral fellow. She has joined Singapore University of Technology and Design (SUTD) as an assistant professor in the pillar of Engineering Product Development since 2014. Her research interests include radiofrequency(RF)/microwave noninvasive, wideband $\mathrm{RF} /$ microwave components, wireless power transfer, $\mathrm{RF}$ aspects of MRI, low-field MRI, and non-linear MRI image reconstructions

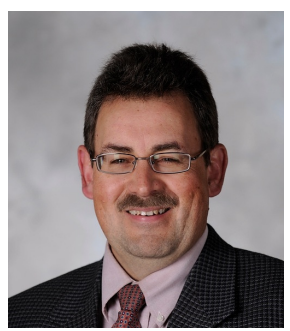

Andrew F. Peterson (StM`82 M`83, SM`92, F`00) Andrew F. Peterson received the B.S., M.S., and $\mathrm{Ph} . \mathrm{D}$. degrees in Electrical Engineering from the University of Illinois, Urbana-Champaign. Since 1989, he has been a member of the faculty of the School of Electrical and Computer Engineering at the Georgia Institute of Technology, where he is a full Professor. He served as the ECE Associate Chair for Faculty Development from 2002-2016. He teaches electromagnetic field theory and computational electromagnetics, and conducts research in the design of antenna arrays and feed structures, the development of computational techniques for electromagnetic applications, and the application of machine learning to electromagnetic analysis and design. He is a coauthor of the 1998 text Computational Methods for Electromagnetics, the 2016 text Higher-order Techniques in Computational Electromagnetics, several lectures in the Morgan/Claypool Synthesis Library, and approximately 300 journal and conference research publications. He has served as a consultant to industrial organizations in the areas of array antenna design and the development of electromagnetic modeling software.

Dr. Peterson served as the General Chair of the 1998 IEEE AP-S International Symposium and URSI/USNC Radio Science Meeting, as Chair of the IEEE Atlanta Section, as a member of IEEE AP-S AdCom, and as a member of the Board of Directors of the Applied Computational Electromagnetics Society (ACES). He was the President of the IEEE AP-S during 2006, and the President of ACES from 2011 to 2013. He also served as a Track Editor and previously an Associate Editor for the IEEE Transactions on Antennas and Propagation, and served as an Associate Editor for the IEEE Antennas and Wireless Propagation Letters. He was the Technical Program Co-Chair for the 2009 ACES Conference in Monterey and the 2019 IEEE AP/URSI Symposium in Atlanta, and will be the TPC Chair for the 2022 IEEE AP/URSI Symposium in Denver. He is a Fellow of the IEEE, a Fellow of ACES, and a recipient of the IEEE Third Millennium Medal. He is also a member of the International Union of Radio Scientists (URSI) Commission B, the American Society for Engineering Education, and the American Association of University Professors. 